\title{
Sequence analysis and spatiotemporal developmental distribution of the Cat-1-type transporter slc7a1a in zebrafish (Danio rerio)
}

\author{
Ståle Ellingsen • Shailesh Narawane • Anders Fjose • \\ Tiziano Verri • Ivar Rønnestad (1)
}

Received: 22 May 2020 / Accepted: 1 September 2020/Published online: 27 September 2020

(C) The Author(s) 2020

\begin{abstract}
Cationic amino acid transporter 1 (Cat-1 alias $\mathrm{Slc7a1)}$ is a $\mathrm{Na}^{+}$-independent carrier system involved in transport and absorption of the cationic amino acids lysine, arginine, histidine, and ornithine and has also been shown to be indispensable in a large variety of biological processes. Starting from isolated full-length zebrafish (Danio rerio) cDNA for slc7ala, we performed comparative and phylogenetic sequence analysis, investigated the conservation of the gene during vertebrate evolution, and defined tissue expression during zebrafish development. Whole mount in situ hybridization first detected slc7ala transcripts in somites,
\end{abstract}

Electronic supplementary material The online version of this article (https://doi.org/10.1007/s10695-020-00873-x) contains supplementary material, which is available to authorized users.

S. Ellingsen $\cdot$ S. Narawane $\cdot$ A. Fjose

Department of Molecular Biology, University of Bergen, Postbox 7803, NO-5020 Bergen, Norway

I. Rønnestad $(\bowtie)$

Department of Biological Sciences, University of Bergen, Postbox 7803, NO-5020 Bergen, Norway

e-mail: Ivar.Ronnestad@uib.no

T. Verri

Department of Biological and Environmental Sciences and Technologies, University of Salento, via Prov.le Lecce-Monteroni, I-73100 Lecce, Italy

Present Address.

S. Ellingsen

Department of Biological Sciences, University of Bergen, Postbox 7803, NO-5020 Bergen, Norway eyes, and brain at $14 \mathrm{~h}$ post-fertilization (hpf) with additional expression in the distal nephron at $24 \mathrm{hpf}$ and in branchial arches at 3 days post-fertilization (dpf), with significant increase by $5 \mathrm{dpf}$. Taken together, the expression analysis of the zebrafish Cat-1 system gene slc7ala suggests a functional role(s) during the early development of the central nervous system, muscle, gills, and kidney.

Keywords Cationic amino acid transport(ers) - slc7a $1 a$. $s l c 7 a 1 b \cdot$ Spatiotemporal expression $\cdot$ Zebrafish

\section{Introduction}

The cationic amino acid transporter 1 (Cat-1), a member of the solute carrier (SLC) 7 family of proteins, also referred to as solute carrier family 7 member 1 (Slc7a1), is a $\mathrm{Na}^{+}$-independent transporter of cationic amino acids. In mammals, the Cat protein group consists of Cat-1, Cat-2a, Cat-2b, and Cat-3, all exhibiting a nearly identical substrate specificity for cationic L-amino acids (Deves et al. 1998; Closs et al. 2004; Closs et al. 2006). Cat-4 is acknowledged to have no affinity for cationic, neutral, or acidic amino acids and is referred to as an orphan transporter, similarly to the Cat protein referred to as Slc7a14 (Closs et al. 2006). So far, very little information is available on the molecular and functional characterizations of these transporters in teleost fish $(\mathrm{Gu}$ et al. 2014). 
Cat proteins are predicted to have 14 transmembrane (TM) regions, with intracellular $\mathrm{N}$ - and $\mathrm{C}$-termini (Albritton et al. 1989). CAT proteins show a variable number of amino acids, also due to the occurrence of alternative splicing events, see, e.g., human SLC7A1 (629 amino acids), SLC7A2A (657 amino acids), SLC7A2B (658 amino acids), SLC7A3 (619 amino acids), SLC7A4 (635 amino acids), and SLC7A14 (711 amino acids) (Closs et al. 2006).

Special features of all Cat proteins include a glutamic acid residue $\left(\mathrm{E}^{107}\right)$ located at the intracellular face of the third TM, which is recognized to be necessary for the transport activity of mouse Slc7a1 (Wang et al. 1994). Also, the third extracellular loop of mouse Slc7a1, which acts as an ecotropic murine leukemia virus binding site (Albritton et al. 1993), contains two asparagine residues $\left(\mathrm{N}^{223}\right.$ and $\left.\mathrm{N}^{229}\right)$ that are glycosylated in mouse Slc7a1 (Kim and Cunningham 1993). These N-linked glycosylation sites are conserved in mouse and human proteins (Wang et al. 1996).

Cats mediate bidirectional cationic amino acid transport and support important physiological functions such as protein synthesis and inter-organ amino acid flow (Hatzoglou et al. 2004). Through arginine homeostasis, Cats are also involved in nitric oxide (NO) synthesis, polyamine biosynthesis, and collagen synthesis (Hatzoglou et al. 2004; Closs et al. 2006; North et al. 2009). In addition, in mammals, the SLC7A1/Slc7a1 gene is vital for cell survival during stress as it permits cells to recommence growth as soon as amino acids become available (Hatzoglou et al. 2004; Closs et al. 2006).

Extensive studies in mammalian models have reported that SLC7A1 is associated with endothelial NOS (eNOS) and caveolin in pulmonary artery endothelial cells (McDonald et al. 1997) and that it is localized to the basolateral membrane in polarized Madin-Darby canine kidney (MDCK) and human embryonic kidney 293 (HEK293) cells (Cariappa et al. 2002; Kizhatil and Albritton 2002). Human keratinocytes constitutively express $S L C 7 A 1$ and SLC7A2 that mediate arginine influx essential for both inducible NOS (iNOS) and arginase enzyme activities, which eventually modulate proliferation and differentiation of epidermal skin cells (Schnorr et al. 2003). In addition, SLC7A1 is expressed in the brain microvascular endothelial cells at the human blood brain barrier (Umeki et al. 2002), and it localizes in retinal capillary endothelial cells where it facilitates arginine transport at the inner blood retinal barrier
(Tomi et al. 2009). Functionally, SLC7A1-mediated arginine import is essential for both differentiation and proliferation of erythrocytes (Shima et al. 2006). Slc7a1 loss-of-function in mice leads to anemia (Perkins et al. 1997) and $25 \%$ size reduction compared with wild-type littermates and perinatal death (Nicholson et al. 1998). Overall, SLC7A1/Slc7a1 distributes differentially among different cells, with varying affinities for basic amino acids arginine, lysine, and ornithine (MacLeod 1996; Closs et al. 2004). Little information is available on changes in SLC7A1/Slc7al expression during embryonic development. Notably, amino acid transport promotes preimplantation mouse embryo development at different stages, e.g., nonessential amino acid transport improves development mainly during cleavage, while essential amino acid transport supports development after the eight-cell stage (Van Winkle 2001).

In this context and with special regard to the elucidation of the role of basic amino acid transport processes in teleost systems and compartments, where at least to our knowledge no information is available to date, we isolated the full-length slc7ala in zebrafish (Danio rerio) and focused on its spatial expression during the early stages of development. Our findings revealed conservation of functionally important amino acids in the zebrafish slc7ala sequence along with the conservation of synteny, which suggests a common biochemical scheme in basic amino acid transport processes across teleost fish membranes, and from teleost fish to mammals. Also, in situ hybridization revealed embryonic and larval stage specific slc7ala expression in eyes, somites, distal nephrons, and branchial arches. Taken together, these results suggest that slc7ala has embryonic stage and organ specific expression and is possibly important for normal embryonic development and function.

\section{Materials and methods}

\section{Zebrafish maintenance}

Zebrafish were maintained and bred at HIB, University of Bergen, as described elsewhere (Stuart et al. 1988). Zebrafish embryos were obtained from natural mating, and pigmentation was prevented by adding $0.003 \%$ phenylthiourea (PTU) to $\mathrm{E} 3$ medium $(5 \mathrm{mM} \mathrm{NaCl}$, $0.17 \mathrm{mM} \mathrm{KCl}, 0.33 \mathrm{mM} \mathrm{CaCl}_{2}, 0.33 \mathrm{mM} \mathrm{MgSO}_{4}$ ). 
Sequence analysis

The nucleotide and protein sequences described in this study were obtained from Ensembl (http://www.ensembl. org/). Predicted transcripts used for slc7ala isolation refer to Ensembl Transcript ID: ENSDART00000008248 (Ensembl Gene ID: ENSDARG00000016439). The cloned zebrafish slc7ala was sequenced and the translated sequence used for sequence comparisons.

The SLC7A1/Slc7a1-type amino acid sequences used for sequence comparison are in Table S1 (see also Appendix I in Supplementary Material). Multiple protein sequence alignments were performed using Clustal Omega (https://www.ebi.ac.uk/Tools/msa/clustalo/). The neighbor-joining $(\mathrm{NJ})$ method-based phylogenetic tree was built using MEGA X (http://www.megasoftware. net).

Putative transmembrane domains were predicted using TMHMM 2.0 (http://www.cbs.dtu. $\mathrm{dk} /$ services/TMHMM/), which is part of the Simple Modular Architecture Research Tool (SMART) (http://smart.embl-heidelberg.de/). Potential $\mathrm{N}$ glycosylation and protein kinase $\mathrm{C}$ recognition sequences were identified using the PROSITE 19.7 computational tools (http://www.expasy.org/prosite/).

Conservation of the zebrafish slc7ala gene with respect to other vertebrate orthologous/paralogous genes (synteny) was evaluated by gene database consulting at the National Center for Biotechnology Information (NCBI) (https://www.ncbi.nlm.nih.gov/gene).

\section{Sequence isolation}

A slc7ala cDNA was cloned from total RNA retrotranscription followed by PCR amplification (as described previously; Rønnestad et al. 2010) using a specific primer pair encompassing the open reading frame (ORF) (for details, see Fig. $1 \mathrm{a}$ and $\mathrm{b}$; for primers, see also Table S2). The in situ probes for slc7ala were prepared by PCR amplification of a slc7ala cDNA fragment (for details, see Fig. 1 a and b; for primers, see also Table S2). The PCR products were cloned into a pCRII-TOPO vector (Invitrogen, Germany). All sequences were verified by sequencing.

Protein modeling

Phyre $^{2}$ (Kelley et al. 2015) in intensive mode (http://www.sbg.bio.ic.ac.uk/phyre2/html/page.cgi?id= index) was used to predict the structures of the zebrafish Slc7a1a proteins (isoforms X1 and X2) from their sequences (for details, see Fig. 1c; see also Appendix I in Supplementary Material). Analogously, Phyre $^{2}$ was used to predict the structures of all the SLC7-type proteins used for comparison (for details, see Table 1 and Fig. 4c; see also Appendix I in Supplementary Material). Whenever required, pairwise structure alignments were generated by TM-align (Zhang and Skolnick 2005) at https://zhanglab.ccmb.med.umich. edu/TM-align/. YASARA View (Krieger and Vriend 2014) was used to visualize all the various threedimensional structures.

RNA probe preparation and DIG-labeling

Plasmid DNA was linearized with appropriate restriction endonucleases for $5 \mathrm{~h}$ at $37{ }^{\circ} \mathrm{C}$, purified using QIAquick Nucleotide Removal Kit (Qiagen, Hilden, Germany), and the degree of linearization was examined on a $1 \%$ agarose gel. In vitro transcription to produce digoxigenin (DIG)-labeled RNA probe was carried out combining linearized plasmid, $1 \mu \mathrm{g}$ DIG labeling mix (Roche, Mannheim, Germany), $2 \mu$ transcription buffer, $2 \mu$ l RNase inhibitor (Roche, Mannheim, Germany), $1 \mu 1$ T7/Sp6 RNA polymerase (Roche), and $2 \mu$ l RNasefree $\mathrm{ddH}_{2} \mathrm{O}$ to a final volume of $20 \mu$. The mix was incubated at $37^{\circ} \mathrm{C}$ for $2 \mathrm{~h}$. This was followed by DNase I treatment for $15 \mathrm{~min}$ at $37{ }^{\circ} \mathrm{C}$. Labeled RNA was purified using the RNeasy Mini Kit (Qiagen, Hilden, Germany); probe length was verified by agarose gel and then dissolved in $150 \mu$ hybridization buffer and stored at $-20{ }^{\circ} \mathrm{C}$ until use.

In situ hybridization

Zebrafish embryos, collected at $24 \mathrm{hpf}, 3 \mathrm{dpf}$, and $5 \mathrm{dpf}$, were dechorionated, anesthetized with tricaine, washed with E3, and fixed overnight in $4 \%$ paraformaldehyde (PFA) at $4{ }^{\circ} \mathrm{C}$. Twelve-fourteen hours post-fertilization embryos were fixed in 4\% PFA before dechorionation. Pigmentation was prevented using $0.003 \%$ PTU in E3 medium. Fixed embryos were placed in $100 \%$ methanol at $-20^{\circ} \mathrm{C}$ until use.

Whole mount in situ hybridization was carried out as described previously (Seo et al. 1998). Briefly, the DIGlabeled sense and antisense zebrafish slc7al RNA probes were used, the former as negative control. 
Imaging

In situ hybridization images were captured with Leica M420 ${ }^{\mathrm{TM}}$ and Nikon EPI-FL3 ${ }^{\mathrm{TM}}$ microscope equipped with micropublisher 5.0 RTV camera (QImaging). Figures were generated using Adobe CS2 Photoshop ${ }^{\mathrm{TM}}$ and Illustrator ${ }^{\mathrm{TM}}$.

\section{Ethical treatment of animals}

Zebrafish were maintained and experiments conducted in compliance with the Norwegian Animal Welfare Act guidelines. No ethical permission was needed. According to the EU Directive 2010/63/EU on the protection of animals used for scientific purposes, implemented in Norwegian legislation as of December 12, 2014, early life stages of zebrafish are not protected as animals until the stage of being capable of independent feeding, i.e., 5 days post fertilization (dpf).

\section{Results}

Cat-1 conservation among vertebrates

Detailed sequence analysis and interspecies comparison among vertebrates were performed to ascertain the identity of the cloned zebrafish Cat-1-type transporter gene and its organization. As assessed by GenBank database consulting (December 2019), two slc7al-type genes are present in the zebrafish genome (GRCz11 Assembly) (Table 1), namely slc7ala (also known as slc7al; the gene analyzed in this study) and slc7alb (also known as zgc:63694). Both genes produce "predicted" alternative splicing mRNA forms. In the case of slc7ala, there are four predicted transcript variants in GenBank, indicated as X1 (Acc. N. XM_678531.7), X2 (Acc. N. XM_021479384.1), X3 (Acc. N. XM_005155277.4), and X4 (Acc. N. XR_002459600.1), and three predicted transcript variants in Ensembl, indicated as -201 (Transcript ID ENSDART00000008248.9), -202 (Transcript ID ENSDART00000146370.3), and -203 (Transcript ID ENSDART00000183510.1). These converge into two predicted alternative amino acid sequences in GenBank, indicated as isoforms X1 (e.g., Acc. N. XP_683623.4) and X2 (e.g., XP_005155334.1), and into three predicted alternative amino acid sequences in Ensembl, indicated as -201 (Protein ID ENSDARP00000027285.8), -202 (Protein ID
Fig. 1 Nucleotide and predicted amino acid sequence of zebrafish slc7ala. The figure was generated using ORFfinder (https://www. ncbi.nlm.nih.gov/orffinder/). Numbers on the left refer to the nucleotide (upper row) and amino acid (lower row) positions. Nucleotides are numbered, starting from the first ATG initiation codon. Asterisk indicates the stop codon. The specific primers used for full-length cDNA cloning and whole mount in situ hybridization probe generation (see also Table S2) are indicated in green and orange, respectively. In the amino acid sequence, putative transmembrane domains, obtained using the TMHMM v. 2.0 program as implemented in SMART, are indicated by arrows and named 1 to 14 . Potential extracellular N-glycosylation sites (white boxes) and potential protein kinase $\mathrm{C}$ phosphorylation sites at the cytoplasmic surface (dark gray boxes) were obtained using the ScanProsite tool. Coding exons are drawn in black and blue. The alternatively spliced forms of coding exon 6 (exon 6 a and exon 6b) are drawn in italics. a Slc7a1a isoform X1. b Slc7a1a isoform X2. c Three-dimensional appearance (lateral view) of zebrafish Slc7a1a proteins (isoform X1 and isoform X2). Homology modeling by Phyre $^{2}$ (intensive mode) was used to predict the structures. The structure of a proton-coupled amino acid transporter with the leucine transporter (LeuT) fold from Geobacillus kaustophilus (GkApcT) (Protein Data Bank Acc. No. 5OQT) was the template. For both isoforms, confidence (i.e. , the probability that the match between the query sequence and the template is a true homology and thus that the template is correct) reached the value of $100 \%$ (the highest accuracy) and the residues in the model covered $71 \%$ of the corresponding experimental structure. After pairwise structure alignment (TM-align), the superimposed structures (i.e., GkApcT vs. Slc7a1a isoform X1 or Slc7a1a isoform X2) were drawn (YASARA View). Putative transmembrane domains are named 1 to 12 (please note that transmembrane domains 1 and 6 are indicated as $1 \mathrm{a}$ and $1 \mathrm{~b}$ and $6 \mathrm{a}$ and $6 \mathrm{~b}$, respectively). For each protein, the region of amino acid sequence change due to the alternative splicing spans from approximately the second half of transmembrane domain 8 to the first half of transmembrane domain 9 through a cytoplasmic beta hairpin loop (delimited by a dashed line)

ENSDARP00000121637.1), and -203 (Protein ID ENSDARP00000152628.1) (Fig. 1a, b; Table S1). The cloned full-length cDNA did code for the slc7ala mRNA indicated as transcript variant $\mathrm{X} 3$ and coding for the Slc7a1a protein indicated as isoform X2 (Fig. 1b). In particular, the cloned zebrafish slc7ala cDNA was 2081 nucleotides long, with an ORF of $1950 \mathrm{nu}-$ cleotides coding a putative protein of 650 amino acids (Fig. 1b). Hydropathy analysis predicted 14 potential transmembrane domains with a large intracellular loop between transmembrane domains 10 and 11 (Fig. 1a and b). Such hydropathy findings were largely confirmed by a parallel analysis of the three-dimensional structures of zebrafish Slc7A1a (isoform X1 and isoform X2) proteins generated by homology modeling on a protoncoupled amino acid transporter with the leucine 
$-5$

1 ATGGTTTTGAAAAAGCTTCTGCGTTTCGGGAAACAGCTGCTGAGA $\begin{array}{lllllllllllllllll}1 & M & V & L & K & K & \text { L } & \text { L } & R & F & G & K & Q & L & L & R\end{array}$

46 GTAAAGGTTGTAAACTGCAACTCAGAGGAATCGCGACTGTCCCGA $16 \quad V \quad K \quad V \quad V \quad N \quad C \quad N \quad S \quad E \quad E \quad S \quad R \quad L \quad S \quad R$

91 TGTCTGAACACTTTTGACCTGGTGGCTCTTGGTGTGGGCAGTACA $\begin{array}{llllllllllllllll}31 & C & L & N & T & F & D & L & V & A & L & G & V & G & S & T\end{array}$ L-1

136 TTAGGAGCAGGGGTTTATGTCCTGGCCGGAGCTGTGGCTCGAGAA $\begin{array}{llllllllllllllll}46 & L & G & A & G & V & Y & V & L & A & G & A & V & A & R & E\end{array}$ L

181 AACGCCGGACCGGCCATTGTGCTGTCGTTTCTCATCGCCGCTCTG 61 N A G P A I V L S F L I A A L

226 GCTTCAGTGCTCGCTGGTCTCTGTTATGCAGAGTTTGGAGCCAGA $\begin{array}{llllllllllllllll}76 & A & S & V & L & A & G & L & C & Y & A & E & F & G & A & R\end{array}$ A S $V$ L

271 GTGCCCAAAACAGGATCAGCGTACCTGTACAGCTACGTGACTGTA

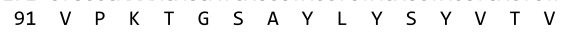
A

316 GGAGAGCTGTGGGCCTTTATAACTGGCTGGAACCTGATTCTTTCA $\begin{array}{llllllllllllllll}106 & G & E & L & W & A & F & I & T & G & W & N & L & I & L & S\end{array}$ G-3-10.-1

361 TATGTTATAGGTACCTCAAGTGTTGCCCGAGCTTGGAGTGCCACA $\begin{array}{llllllllllllllll}121 & Y & V & I & G & T & S & S & V & A & R & A & W & S & A & T\end{array}$

406 TTTGATGAGTTGATTGGAAAACATATTGAGCATTTCTGCCGCCAG $\begin{array}{llllllllllllllll}136 & F & D & E & L & I & G & K & H & I & E & H & F & C & R & Q\end{array}$

451 TACATGTCCATGAATGCGCCGGGCGTCCTGGCGGAGTATCCAGAT $\begin{array}{llllllllllllllll}151 & Y & M & S & M & N & A & P & G & V & L & A & E & Y & P & D\end{array}$

496 ATGTTTTCTGTTTTCATCATCCTCACACTCACAGGCCTGCTGGCC 166 M F $\quad$ S $V$ V F

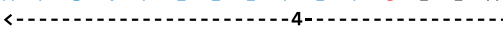

541 TTTGGGGTGAAGGAATCAGCCATGGTCAATAAAGTGTTCACCTGC 181 F $G \quad V \quad K \quad E \quad S \quad A \quad M \quad V \quad N \quad K \quad V \quad F \quad T \quad C$ -10-1

586 ATCAACATTCTGGTGCTGCTGTTCATGGTCGTCTCTGGACTAGTC $\begin{array}{llllllllllllllll}196 & \text { I } & N & \text { I } & \text { L } & V & \text { L } & \text { L } & F & M & V & V & S & G & \text { L } & \text { V }\end{array}$

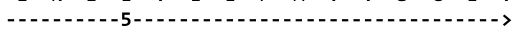

631 AAAGGGACTCTGAAGAACTGGCACCTGGACCCCGATGAGATCCTC

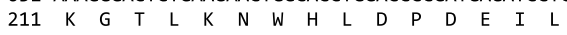

676 AATGCAACCAACTCCACCCTTAATGCCACGCAGCCGCTGCCATCA

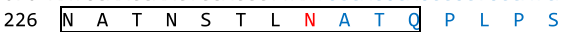

721 GAAGAGATGCTGGGTCAAGGTGGCTTCATGCCTTTCGGCTTCACA

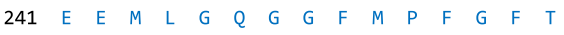
(3)

766 GGGGTTTTATCAGGAGCGGCGACCTGCTTTTACGCCTTCGTTGGC

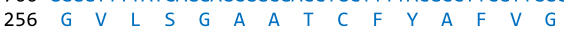

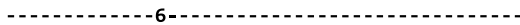

811 TTCGACTGCATCGCAACTACAGGTGAGGAGGTAAAAAACCCTCAA

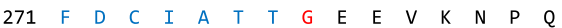
$->$

856 CGAGCCATTCCCATCGGCATCGTCTCCTCGCTCCTCATCTGCTTT $\begin{array}{llllllllllllllll}286 & R & A & I & P & I & G & I & V & S & S & L & L & I & C & F\end{array}$

901 GTGGCGTATTTTGGTGTATCAGCCGCGCTCACCATGATGATGCCC

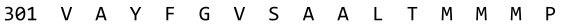
-

946 TATTACATGCTGGATAAAAACAGTCCTCTTCCTGTGGCGTTTAAG

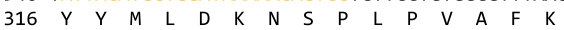

991 TATGTTGGTTGGGAAGGAGCTACATATGCTGTGGCTGTAGGGTCT $\begin{array}{llllllllllllllll}331 & Y & V & G & W & E & G & A & T & Y & A & V & A & V & G & S\end{array}$ a

1036 CTCTGCGCCCTGTCCACCAGCCTGCTTGGCGCCATGTTCCCCATG

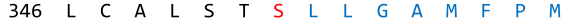

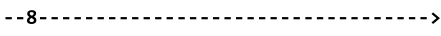

1081 CCCCGTGTCCTGTGGGCCATGGCTGACGATGGCCTGCTCTTCAAA

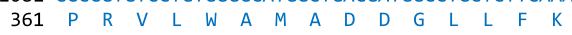

1126 TTCATGGCTGGGATCAGCGAGAGAACAAAAACACCCATAAAAGCC $\begin{array}{llllllllllllllll}376 & F & M & A & G & I & S & E & R & T & K & T & P & I & K & A\end{array}$ <-1

1171 ACTATAATGTCTGGCTTTCTCGCAGCAATCATGGCTTTCCTGTTT

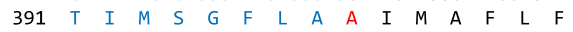
-

1216 GACCTGAAGGACTTGGTTGATCTAATGTCTATAGGGACTCTTCTG $\begin{array}{llllllllllllllll}406 & D & L & K & D & L & V & D & L & M & S & I & G & T & L & L\end{array}$ <

1261 GCCTACACACTGGTTGCTGCCTGCGTTCTTGTACTCAGGTACCAG $\begin{array}{llllllllllllllll}421 & \text { A } & Y & T & \text { L } & V & \text { A } & \text { A } & C & V & \text { L } & V & \text { L } & R & Y & Q\end{array}$ -10-10

1306 CCGGAGCAGTTTTCTCAAACATATCACATAGCCAACACACATGAA $\begin{array}{llllllllllllllll}436 & P & E & Q & F & S & Q & T & Y & H & I & A & N & T & H & E\end{array}$

1351 GACATGGAAATGAGTGAAACAATAAGCACACCCAGCATGGGGATC

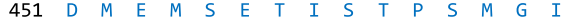

1396 CTGCCCGGCGTAGAGGAGCGTTTCAGCTTCAAAAACCTGCTCTTT $466 L \quad P \quad G \quad V \quad E \quad E \quad R \quad F \quad S F G \quad N \quad L \quad L \quad F$

1441 CCGGACATCATCGAACCCTCCAACCTGTCTGGATTCACCGTTAAC

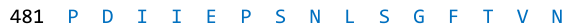

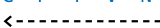

1486 ATCTGCACCAGTCTGCTCGGTCTGCTGATTCTCAGCTTCAGTCTG $\begin{array}{llllllllllllllllll}496 & \text { I } & C & T & S & L & L & G & \text { L } & \text { L } & \text { I } & \text { L } & \text { S } & F & \text { S } & \text { L }\end{array}$ -

1531 TTGGCGGTTCGGGGAGGAATAGCGAGCTGGAATATCATCACTCTC $\begin{array}{rccccccccccccccc}511 & L & A & V & R & G & G & I & A & S & W & N & I & I & T & L\end{array}$

1576 GCTGTCCTGTTCGGCCTGTGTGTCATTGTCACATTTATCATCTGG $\begin{array}{llllllllllllllll}526 & \text { A } & V & \text { L } & F & G & \text { L } & C & V & \text { I } & \text { V } & \text { T } & \text { F } & \text { I } & \text { I } & \text { W }\end{array}$ - 12-1

1621 AGGCAACCTGAGAGTAAAACCAAGCTCTCTTTTAAGGTTCCTTGT $\begin{array}{lllllllllllllllll}541 & R & Q & P & E & S & K & T & K & L & S & F & K & V & P & C\end{array}$

1666 CTTCCCTTCATTCCGGTGGTGAGCATGTTTGTCAACGTCTACCTG $\begin{array}{llllllllllllllllll}556 & L & P & F & I & P & V & V & S & M & F & V & N & V & Y & L\end{array}$ -

1711 ATGATGCAACTGGACAGAGGCACCTGGATACGATTTGCCATCTGG $\begin{array}{llllllllllllllll}571 & M & M & Q & L & D & R & G & T & W & I & R & F & A & I & W\end{array}$ -->

1756 ATGTCTATAGGACTCGTAATCTACTTTGGCTATGGGATCTGGCAC $\begin{array}{lllllllllllllllll}586 & M & S & I & G & L & V & I & Y & F & G & Y & G & I & W & H\end{array}$ -14-14

1801 AGCACCGAAGCCGCTCTGGCTCATTCCAGTATGGACGAAGAGTTG 601 S T

1846 AGTGTGTACAAACCTGCCTGCGGTCTCAACCGAGACAGCGTGACC $\begin{array}{rlllllllllllllll}616 & S & V & \text { Y } & \text { K } & \text { P } & \text { A } & C & G & \text { L } & \text { N } & \text { R } & \text { D } & \text { S } & \text { V } & \text { T }\end{array}$

1891 CCAGAGAAAGAGGCTTTCCTCTGTAATGGCCACGGGTCACAGGTA $\begin{array}{llllllllllllllll}631 & P & E & K & E & A & F & L & C & N & G & H & G & S & Q & V\end{array}$

1936 GATGACGATGGAGATATCTAAatgagcaacagtgttatgttgtat

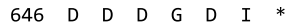

1981 actcagactttacagaaagcactccgtcaggaggtagtgaggaac 2026 acgggtcgctggagtctaatctggtagagctcgatgactgattta 2071 accaatgtc 


\section{$-5$}

cagca

1 ATGGTTTTGAAAAAGCTTCTGCGTTTCGGGAAACAGCTGCTGAGA $\begin{array}{lllllllllllllllll}1 & M & V & L & K & K & L & L & R & F & G & K & Q & L & L & R\end{array}$

46 GTAAAGGTTGTAAACTGCAACTCAGAGGAATCGCGACTGTCCCGA

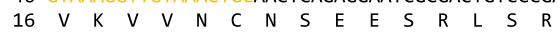

91 TGTCTGAACACTTTTGACCTGGTGGCTCTTGGTGTGGGCAGTACA $\begin{array}{llllllllllllllll}31 & C & L & N & T & F & D & L & V & A & L & G & V & G & S & T\end{array}$ <-1

136 TTAGGAGCAGGGGTTTATGTCCTGGCCGGAGCTGTGGCTCGAGAA $\begin{array}{llllllllllllllll}46 & L & G & A & G & V & Y & V & L & A & G & A & V & A & R & E\end{array}$ L

181 AACGCCGGACCGGCCATTGTGCTGTCGTTTCTCATCGCCGCTCTG 61 N A G P A I V L S F L I A A L

226 GCTTCAGTGCTCGCTGGTCTCTGTTATGCAGAGTTTGGAGCCAGA $\begin{array}{llllllllllllllll}76 & A & S & V & L & A & G & L & C & Y & A & E & F & G & A & R\end{array}$ A

271 GTGCCCAAAACAGGATCAGCGTACCTGTACAGCTACGTGACTGTA

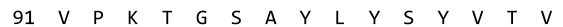
A

316 GGAGAGCTGTGGGCCTTTATAACTGGCTGGAACCTGATTCTTTCA 106 G E L W A F I T G W N L I L S

361 TATGTTATAGGTACCTCAAGTGTTGCCCGAGCTTGGAGTGCCACA $\begin{array}{llllllllllllllll}121 & Y & V & I & G & T & S & S & V & A & R & A & W & S & A & T\end{array}$

406 TTTGATGAGTTGATTGGAAAACATATTGAGCATTTCTGCCGCCAG $\begin{array}{lllllllllllllllll}136 & F & D & E & L & I & G & K & H & I & E & H & F & C & R & Q\end{array}$

451 TACATGTCCATGAATGCGCCGGGCGTCCTGGCGGAGTATCCAGAT $\begin{array}{llllllllllllllll}151 & Y & M & S & M & N & A & P & G & V & L & A & E & Y & P & D\end{array}$

496 ATGTTTTCTGTTTTCATCATCCTCACACTCACAGGCCTGCTGGCC

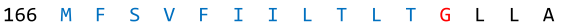

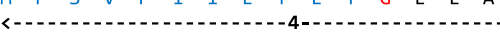

541 TTTGGGGTGAAGGATCAGCCATGGTCAATAAAGTGTTCACCTGC 181 F $G \quad V \quad K \quad E \quad S \quad A \quad M \quad V \quad N \quad K \quad V \quad F \quad T \quad C$ -.-.-.

586 ATCAACATTCTGGTGCTGCTGTTCATGGTCGTCTCTGGACTAGTC $\begin{array}{llllllllllllllll}196 & \text { I } & N & \text { I } & L & V & L & L & F & M & V & V & S & G & L & V\end{array}$ -

631 AAAGGGACTCTGAAGAACTGGCACCTGGACCCCGATGAGATCCTC $\begin{array}{llllllllllllllll}211 & K & G & T & L & K & N & W & H & L & D & P & D & E & I & L\end{array}$

676 AATGCAACCAACTCCACCCTTAATGCCACGCAGCCGCTGCCATCA

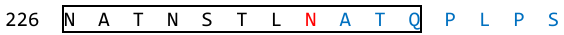

721 GAAGAGATGCTGGGTCAAGGTGGCTTCATGCCTTTCGGCTTCACA

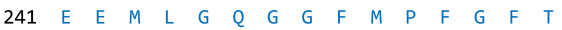
(1)

766 GGGGTTTTATCAGGAGCGGCGACCTGCTTTTACGCCTTCGTTGGC 256 G $V$ L $S$ G A A T C F F Y A $F$ F $V$ G

811 TTCGACTGCATCGCAACTACAGGTGAGGAGGTAAAAAACCCTCAA $\begin{array}{lllllllllllllllll}271 & F & D & C & I & A & T & T & G & E & E & V & K & N & P & Q\end{array}$ $\rightarrow$

856 CGAGCCATTCCCATCGGCATCGTCTCCTCGCTCCTCATCTGCTTT

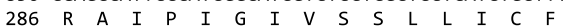
i

901 GTGGCGTATTTTGGTGTATCAGCCGCGCTCACCATGATGATGCCC

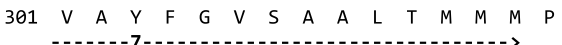

946 TATTACATGCTGGATAAAAACAGTCCTCTTCCTGTGGCGTTTAAG

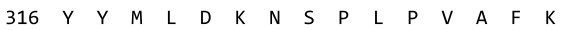

991 TATGTTGGTTGGGAAGGAGCTACATATGCTGTGGCTGTAGGGTCT $\begin{array}{llllllllllllllll}331 & Y & V & G & W & E & G & A & T & Y & A & V & A & V & G & S\end{array}$

Fig. 1 (continued) b

1036 CTCTGCGCCCTGTCCACCAGTCTCTTGGGGTCCTTGTTCCCCCTC

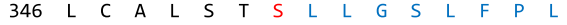
(1)

1081 CCACGTATTATCTTTGCCATGGCCCGGGACGGTCTGCTCTTCTCG $\begin{array}{llllllllllllllll}361 & P & R & I & I & F & A & M & A & R & D & G & L & L & F & S\end{array}$

1126 TTTCTGGCTCGGGTGAGCGAGAAGAAGACGCCCGTCGTGTCCACT

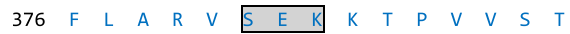
(-)-

1171 CTGGCCTCTGGGGTCACTGCTGCAATCATGGCTTTCCTGTTTGAC $\begin{array}{llllllllllllllll}391 & L & A & S & G & V & T & A & A & I & M & A & F & L & F & D\end{array}$ (1)

1216 CTGAAGGACTTGGTTGATCTAATGTCTATAGGGACTCTTCTGGCC 406 L $\quad$ K $\quad D \quad L \quad V \quad D \quad L \quad M \quad S \quad I \quad G \quad T \quad L \quad L \quad A$

1261 TACACACTGGTTGCTGCCTGCGTTCTTGTACTCAGGTACCAGCCG $\begin{array}{lllllllllllllllll}421 & Y & T & L & V & A & A & C & V & \text { L } & V & \text { L } & R & Y & Q & P\end{array}$ -10-10

1306 GAGCAGTTTTCTCAAACATATCACATAGCCAACACACATGAAGAC $\begin{array}{llllllllllllllll}436 & E & Q & F & S & Q & T & Y & H & I & A & N & T & H & E & D\end{array}$

1351 ATGGAAATGAGTGAAACAATAAGCACACCCAGCATGGGGATCCTG $\begin{array}{lllllllllllllllll}451 & M & E & M & S & E & T & I & S & T & P & S & M & G & I & L\end{array}$

1396 CCCGGCGTAGAGGAGCGTTTCAGCTTCAAAAACCTGCTCTTTCCG

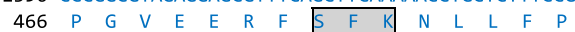

1441 GACATCATCGAACCCTCCAACCTGTCTGGATTCACCGTTAACATC

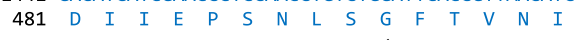
<-- -

1486 TGCACCAGTCTGCTCGGTCTGCTGATTCTCAGCTTCAGTCTGTTG

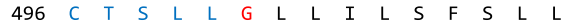
(11-10

1531 GCGGTTCGGGGAGGATAGCGAGCTGGAATATCATCACTCTCGCT

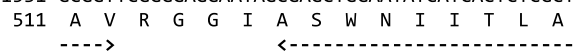
1576 GTCCTGTTCGGCCTGTGTGTCATTGTCACATTTATCATCTGGAGG

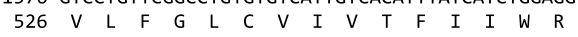
CAACCTGAGATAAACCAAGTCTCTTTAAGGTCTTGT

1621 CAACCTGAGAGTAAAACCAAGCTCTCTTTTAAGGTTCCTTGTCTT $\begin{array}{lllllllllllllllll}541 & Q & P & E & S & K & T & K & L & S & F & K & V & P & C & L\end{array}$ <--

1666 CCCTTCATTCCGGTGGTGAGCATGTTTGTCAACGTCTACCTGATG

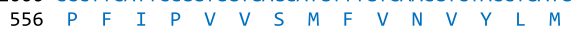
-13-10

1711 ATGCAACTGGACAGAGGCACCTGGATACGATTTGCCATCTGGATG $\begin{array}{llllllllllllllll}571 & M & Q & L & D & R & G & T & W & I & R & F & A & I & W & M\end{array}$$$
\rightarrow
$$

1756 TCTATAGGACTCGTAATCTACTTTGGCTATGGGATCTGGCACAGC

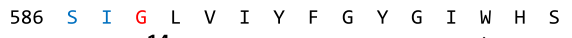
- 14 G V I Y F G Y G I W H

1801 ACCGAAGCCGCTCTGGCTCATTCCAGTATGGACGAAGAGTTGAGT 601 T $E$ A A

1846 GTGTACAAACCTGCCTGCGGTCTCAACCGAGACAGCGTGACCCCA $\begin{array}{cccccccccccccccc}616 & V & Y & K & P & A & C & G & L & N & R & D & S & V & T & P\end{array}$

1891 GAGAAAGAGGCTTTCCTCTGTAATGGCCACGGGTCACAGGTAGAT $\begin{array}{cccccccccccccccc}631 & E & K & E & A & F & L & C & N & G & H & G & S & Q & V & D\end{array}$

1936 GACGATGGAGATATCTAAatgagcaacagtgttatgttgtatact

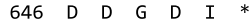

1981 cagactttacagaaagcactccgtcaggaggtagtgaggaacacg 2026 ggtcgctggagtctaatctggtagagctcgatgactgatttaacc 2071 aatgtc 
GkApcT vs. Slc7a1a isoform X1

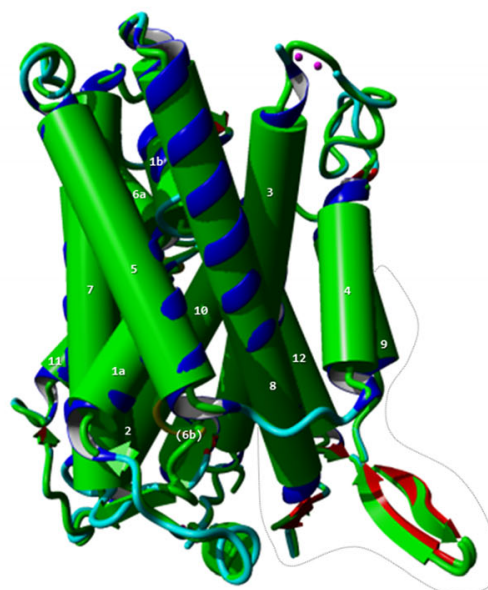

Fig. 1 (continued)

transporter (LeuT) fold from Geobacillus kaustophilus (GkApcT) (Protein Data Bank Acc. No. 5OQT). However, only 12 transmembrane domains occurred in these models, with transmembrane domains 11 and 12 of GkApcT corresponding to transmembrane domains 13 and 14 of the zebrafish Slc7ala proteins (see hydropathy profile in Fig. 1a and b), due to the lack of superimposition in those parts of the zebrafish Slc7ala proteins corresponding to transmembrane domains 11 and 12 . Moreover, comparing zebrafish Slc7a1a isoform X2 to zebrafish Slc7ala isoform X1 revealed differences in the protein sequence (amino acid positions X1:352-399 and X2:352-398) and length (X1: 651 vs. X2: 650 amino acids), due to an alternative splicing event involving coding exons $6 \mathrm{a}$ and $6 \mathrm{~b}$ (Fig. 1a, b, and c; Fig. $\mathrm{S} 1$; for comparison, see also Fig. $4 \mathrm{a}, \mathrm{b}$, and c). Such differences were observed in other teleost fish Slc7a1a proteins analyzed in this study (Fig. S1).

Predicted Slc7a1-type amino acid sequences from zebrafish, Atlantic herring, medaka, fugu rubripes, tropical clawed frog, chicken, cattle, mouse, rhesus monkey, and human were aligned using Clustal Omega, and phylogenetic tree analysis was performed (Fig. 2). The phylogenetic tree construction revealed branching of Slc7al-type proteins from teleost fish species in one cluster while the mammalian species branched in a cluster together with chicken and tropical clawed frog. Coherently, teleost fish Slc7a1a and Slc7alb proteins clustered in two separate branches, suggesting gene duplication occurrence in this lower vertebrate group (Fig. 2). Alignment of the zebrafish
GkApcT vs. Slc7a1a isoform X2

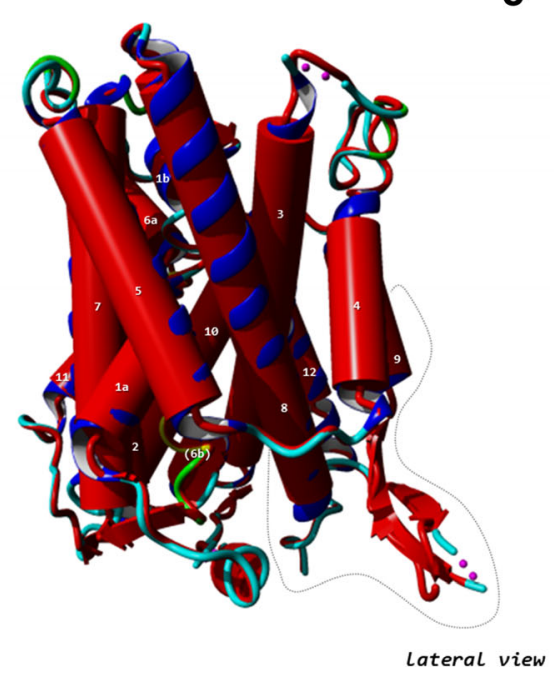

Slc7a1a sequences (Slc7a1a isoform X1: 97.0; Slc7a1a isoform X2: $100 \%$ ) revealed a slightly higher percentage of identity with Slc7ala sequences from the other teleost fish (Atlantic herring 79.4-80.8\%; medaka 78.5-79.8\%; fugu rubripes $80.1 \%$ ) than with any sequences from amphibians (tropical clawed frog Slc7al 68.3\%), birds (chicken SLC7A1 72.1\%), and mammals (cattle SLC7A1 71.0\%; mouse Slc7a1 69.9\%; rhesus monkey and human SLC7A1 70.6\%) (Fig. S2). Moreover, the alignment of the zebrafish Slc7ala sequences revealed high identity percentage with respect to Slc7alb sequences that varied between $\sim 78$ and $\sim 69 \%$ depending on the teleost fish species analyzed (i.e., zebrafish Slc7alb 77.5-77.8\%; Atlantic herring Slc7alb 72.3-72.4\%; medaka Slc7a1b 74.1-75.2\%; fugu rubripes Slc7alb: 68.8-70.3\%).

With respect to relevant protein motifs/regions, putative N-linked glycosylation sites conserved in mouse Slc7a1 and human SLC7A1 (Wang et al. 1996) in the third extracellular loop were - for example - also found in zebrafish Slc7ala and the other teleost fish species studied (Fig. 1a, b; Fig. S1). Again, $\mathrm{E}^{107}$ of mouse Slc7a1, which has been shown to be necessary for the transport activity (Wang et al. 1994), was also conserved in zebrafish Slc7ala and the other species compared (Fig. 1a and b; Fig. S1). Conversely, in teleost fish and amphibians, both the $\mathrm{N}$ - and $\mathrm{C}$-terminal sequences were different than those found in mammals, and, e.g., an RRK motif near to the $\mathrm{N}$-terminal that is conserved in higher vertebrates is replaced by RVK motif in the teleost fish species investigated in this study (Fig. S1). 


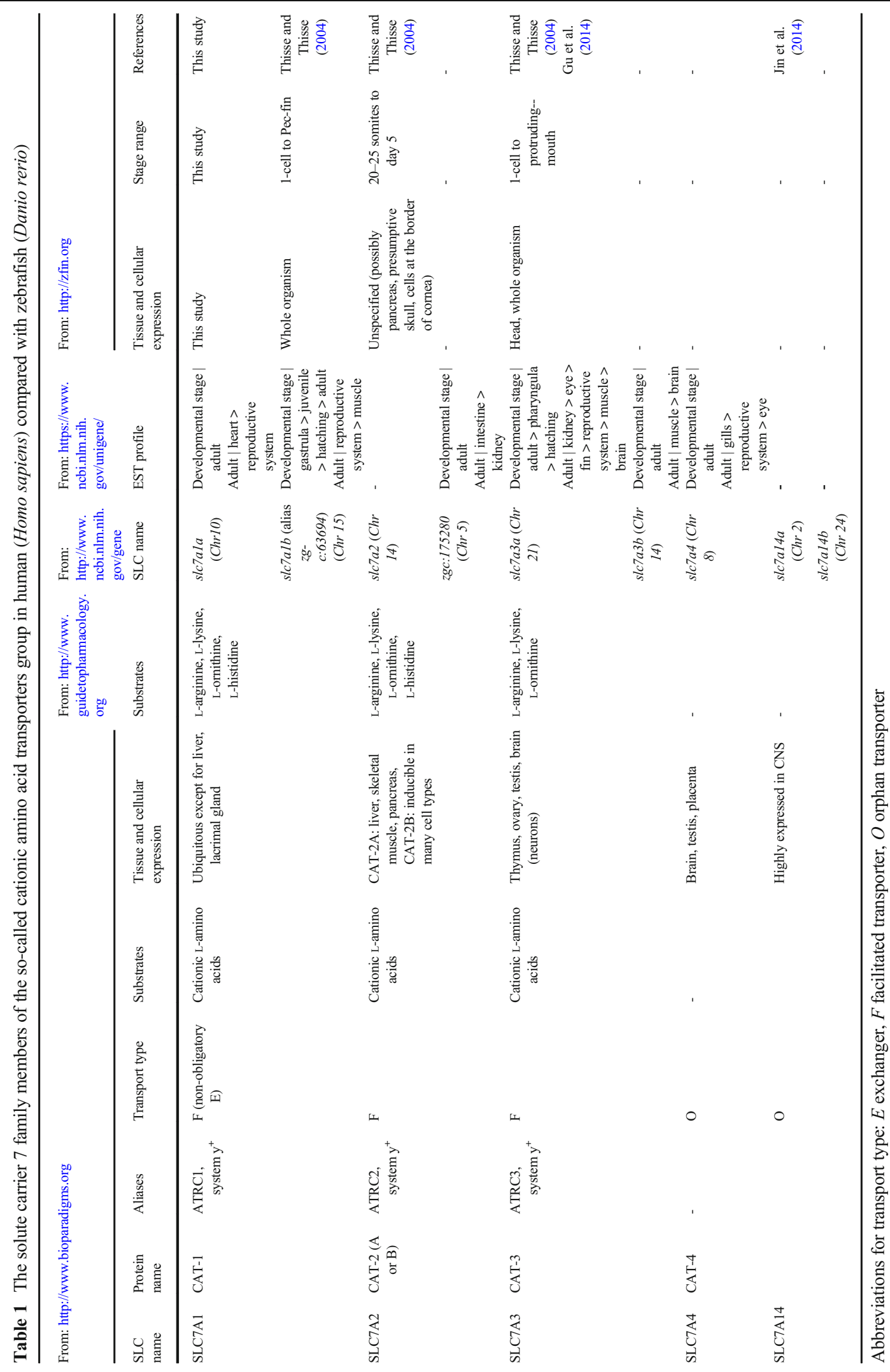




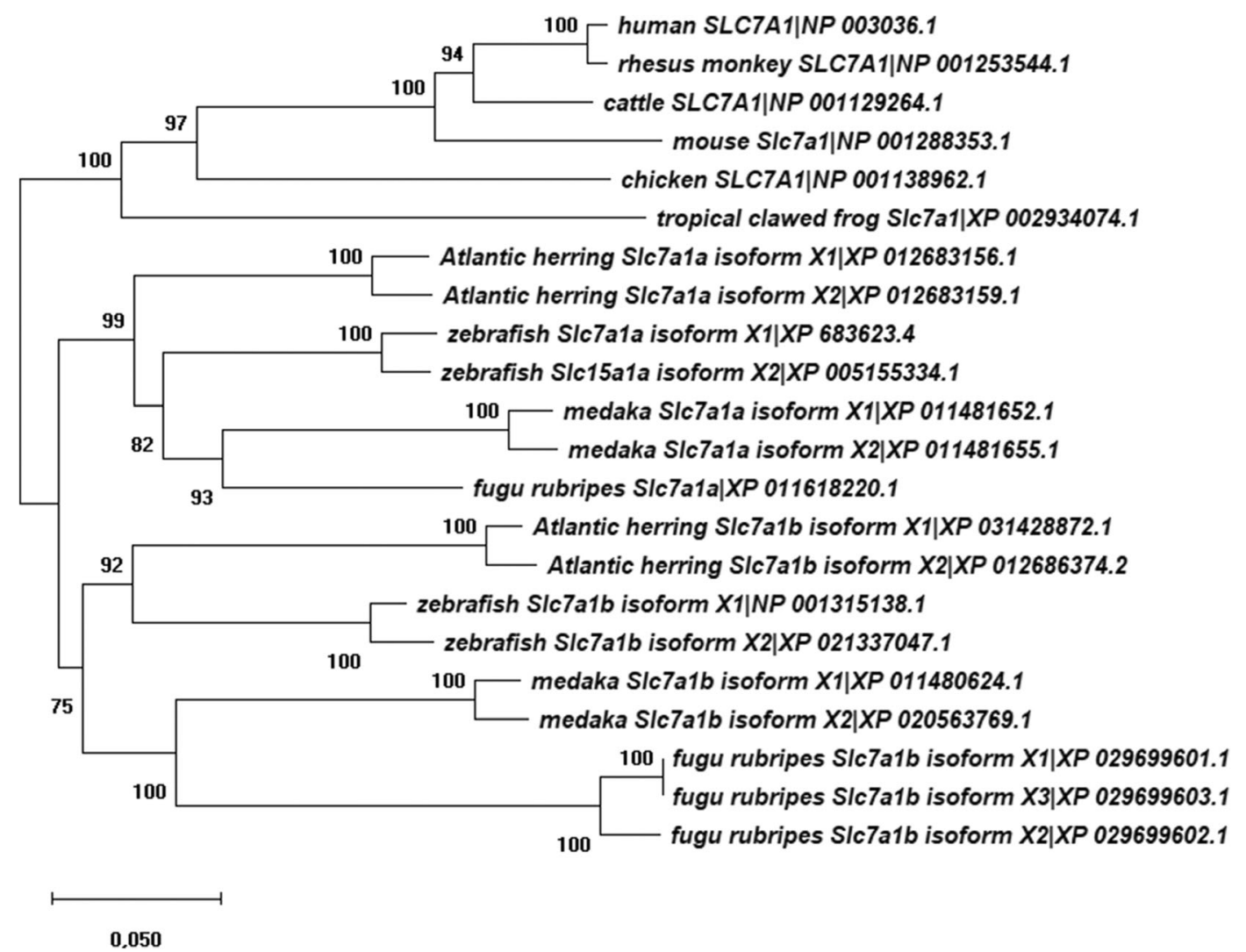

Fig. 2 Slc7a1a evolutionary relationships of taxa. The evolutionary history was inferred using the neighbor-joining method (Saitou and Nei 1987). The optimal tree with the sum of branch length $=157,066,916$ is shown. The percentage of replicate trees in which the associated taxa clustered together in the bootstrap test (1000 replicates) is shown next to the branches (Felsenstein 1985). The tree is drawn to scale, with branch lengths in the same units as those of the evolutionary distances used to infer the phylogenetic

Zebrafish slc7ala localization on chromosome with respect to neighbor genes and in comparison to its orthologues along the vertebrate scale was evaluated by Gene analysis at NCBI, which clearly showed how slc7ala (and not its paralogue slc7alb) lays with muts $2 a$ within a syntenic region common to teleost fish, amphibians, birds and mammals (Table S3).

Taken together, all these gene/genomic findings strongly suggest the concept that zebrafish slc7ala represents the true orthologue of the higher vertebrate slc7a1/Slc7a1/SLC7A1 gene series.

Specific expression during development

Whole-mount in situ hybridization analysis of slc7ala during embryonic development was used to analyze its tree. The evolutionary distances were computed using the Poisson correction method (Zuckerkandl and Pauling 1965) and are in the units of the number of amino acid substitutions per site. The analysis involved 22 amino acid sequences. All positions containing gaps and missing data were eliminated. There were a total of 579 positions in the final dataset. Evolutionary analyses were conducted in MEGA X (Kumar et al. 2018)

expression at various stages of development ranging from $14 \mathrm{hpf}$ to $5 \mathrm{dpf}$. This analysis first detected slc7ala transcripts in somites, optic vesicles, and posterior midbrain around $14 \mathrm{hpf}$ (Fig. 3a). Two distinct stripes (marked by arrows) of slc7ala expression in somites were seen in a dorsal view at $14 \mathrm{hpf}$ (Fig. 3b). More intense staining was observed at $24 \mathrm{hpf}$ (Fig. 3c), with prominent expression in the eyes (Fig. 3d). Additional staining was detected in posterior midbrain, and a thin line of slc7ala expression was observed along the dorsal edges of the rhombomeres at $24 \mathrm{hpf}$ (Fig. 3e). Expression in the somites appeared as broad " $v$ "-shaped stripes with no expression at the somite boundaries (Fig. 3f) that was also seen in a dorsal view as two distinct stripes parallel to the antero-posterior axis along the trunk and tail of the embryo (Fig. 3h). At the same stage, 

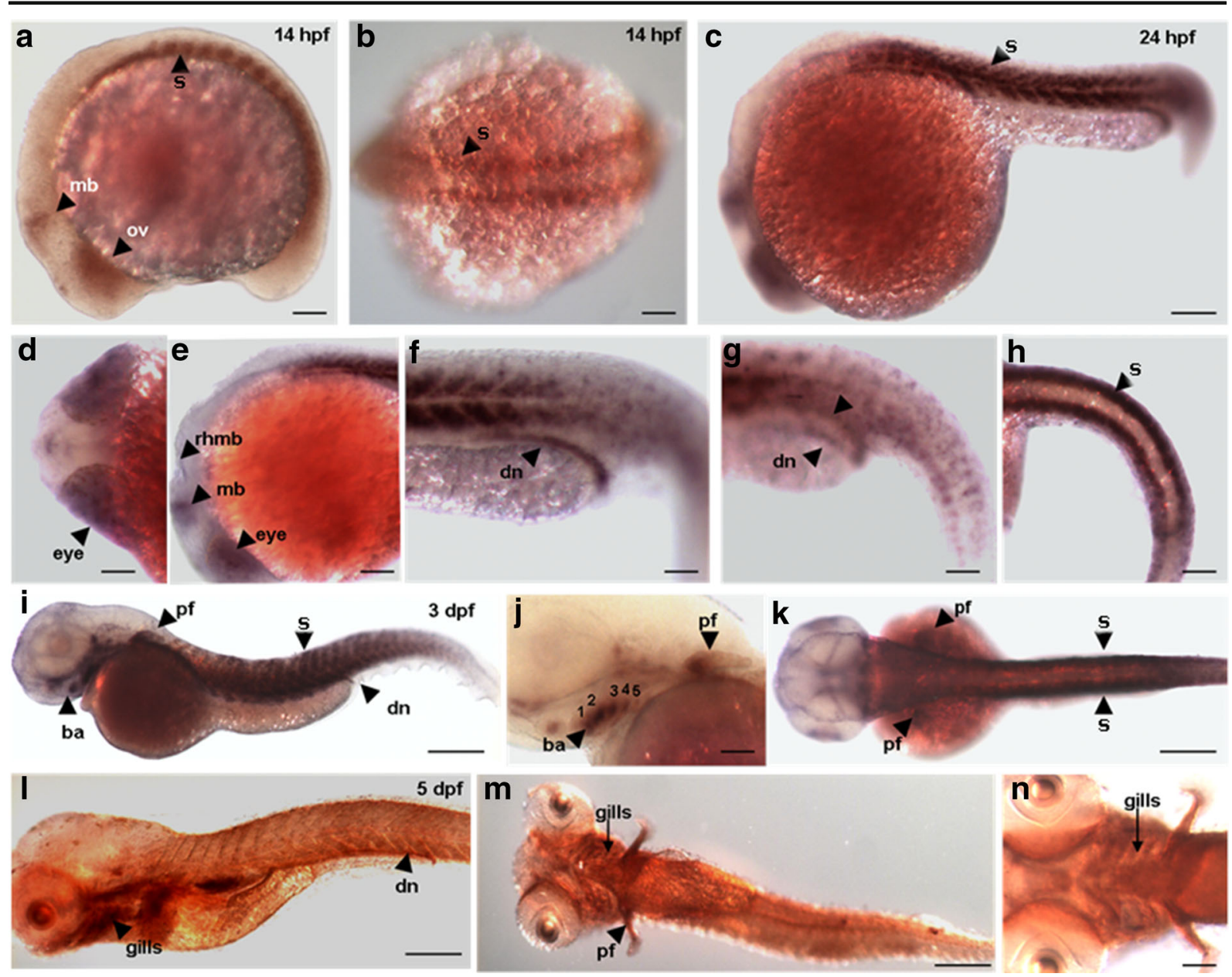

Fig. 3 Spatiotemporal distribution of slc7ala in zebrafish (Danio rerio). Image analysis after whole-mount slc7ala in situ hybridization. a Lateral view shows expression of slc7ala in optic vesicle, midbrain, and somites at $14 \mathrm{hpf}$. b Dorsal view shows slc7ala expression in two stripes of somites at $14 \mathrm{hpf}$. $\mathbf{c}$ Lateral view of slc7ala expression at $24 \mathrm{hpf}$. d Dorsal view of the expression in the eye at $24 \mathrm{hpf}$. e Lateral view shows the expression in the eye, posterior midbrain and dorsal edges of rhombomeres at $24 \mathrm{hpf}$. f Magnified lateral view showing expression in somites and distal nephron at $24 \mathrm{hpf}$. $\mathrm{g}$ Tilted lateral view shows two distal nephrons

slc7ala expression appeared in distal nephrons (Fig. $3 f$ ), which were clearly seen fusing together to form a common opening at the cloaca (arrowheads in Fig. 3g). This expression seems to correspond approximately to the distal late and pronephric duct segments (Wingert et al. 2007). In this respect, slc7ala expression overlaps with both that of slc12a3 in the distal late nephron and that of gata3 in the pronephric duct segments (Wingert et al. 2007). Along with somites and distal nephrons, additional slc7ala expression was observed in the pectoral fins and branchial arches at $3 \mathrm{dpf}$ (Fig. 3i), while (arrows) fusing together at $24 \mathrm{hpf}$. $\mathbf{h}$ Dorsal view focusing on two strips of somites at $24 \mathrm{hpf}$. i Lateral view shows expression in branchial arches, pectoral fin, somites, and distal nephron at $3 \mathrm{dpf}$. j Magnified lateral view with gill arches and pectoral fin buds at 3 dpf. $\mathbf{k}$ Dorsal view showing expression in pectoral fins and somites at $3 \mathrm{dpf}$. I Lateral view at $5 \mathrm{dpf}$. $\mathbf{m}$ Ventral view $5 \mathrm{dpf}$. $\mathbf{n}$ Magnified view of gills at $5 \mathrm{dpf}$. Abbreviations: mb, midbrain; ov, optic vesicle; s, somites; dn, distal nephron, rhmb, rhombomeres; ba, branchial arches; pf, pectoral fin. Scale bar: a, b, d-g, j, n) $50 \mu \mathrm{m} ; \mathbf{c}, \mathbf{h}, \mathbf{i}, \mathbf{k}, \mathbf{m}) 100 \mu \mathrm{m}$

the expression in the eyes at this stage was almost disappeared. Upon close observation, five distinct branchial arches were seen marked by slc7ala expression along with that of the pectoral fins (Fig. 3j). Somite expression was still maintained at $3 \mathrm{dpf}$ (arrowheads in Fig. 3k). At $5 \mathrm{dpf}$, the slc7ala expression was widespread in most parts of the embryo with stronger expression in the gills and distal nephrons (Fig. 31). Five branchial arches forming gills were marked by stronger slc7ala expression along with that in pectoral fins at 5 dpf (Fig. 3m, n). 


\section{Discussion}

Lysine and arginine are important amino acids in metabolism and nutrition. Lysine is essential in animals, i.e., it cannot be synthesized in the body and it needs to be obtained from an external food source. It is important for protein synthesis (growth) and carnitine production; also, it helps the body absorb calcium, and it is required for the formation of collagen, which is crucial for bones and connective tissues including skin, tendon, and cartilage (Civitelli et al. 1992; Flodin 1997; Fini et al. 2001). Arginine is essential for young mammals, while it is conditionally essential for adult mammals, since it is vital in situations such as pregnancy (Bronte and Zanovello 2005), spermatogenesis, maintenance of vascular tone, and hemodynamics (Wu et al. 2009). Arginine is also required for the detoxification of ammonia, which is highly toxic for the central nervous system (North et al. 2009; Wu et al. 2009). The vast majority of teleost fish including zebrafish are ammonotelic, excreting up to $90 \%$ of their nitrogenous waste directly into water as ammonia (Braun et al. 2009a, b).

Amino acid transporters are classified as to belong to the solute carrier (SLC) family of proteins, which consists of more than 400 organic and inorganic carriers (Hediger et al. 2013; Alexander et al. 2019). They transport amino acids over membranes and cells. A large array of amino acid transporters is present on the apical and basolateral membranes of intestinal epithelial cells, absorbing amino acids from the intestinal lumen for subsequent release into the blood (Broer 2008; Kandasamy et al. 2018). Amino acid transporters also have an important function in the vertebrate kidney where they are involved in adjustment of amino acid levels in the ultrafiltrate and final urine (Broer 2008; Kandasamy et al. 2018). In this study, we specifically focused on a zebrafish Cat-1 system, named Slc7ala, which is putatively involved in the transport of lysine, arginine, and cysteine. By detailed sequence analysis, we conferred the structural and sequence specific identity of this zebrafish system, along with its syntenic conservation with evolutionarily related genes, and evidenced its expression during early development.

\section{Zebrafish Slc7a1a amino acid sequence is highly conserved}

To determine identity and specificities of zebrafish Slc7a1a, we performed detailed sequence analysis.
SLC7A1/Slc7a1 proteins from the different vertebrate species analyzed show a variable number of amino acids, e.g., mouse 622, human 629 (Deves and Boyd 1998), as well as rhesus monkey and cattle, chicken 624, while tropical clawed frog exhibited 654 amino acids. Conversely, teleost Slc7a1a proteins show the following amino acids lengths: zebrafish 650-651, Atlantic herring 656-657, medaka 647-648, and fugu rubripes 648, while teleost Slc $7 \mathrm{a} 1 \mathrm{~b}$ proteins exhibit amino acids lengths as follows: zebrafish 646-647, Atlantic herring 654-655, medaka 638-639, and fugu rubripes from 638 to 681-682 depending on the isoform (for details, see Fig. S1). Therefore, zebrafish Slc7a1a and Slc7a1b proteins are more than 20 amino acids longer than mammalian SLC7A1/Slc7a1 proteins, and the length criterion is not a discriminating element to define orthology between SLC7A1/Slc7a1 and Slc7a1a or Slc7a1b transporters.

Analogously, SLC7A1/Slc7a1 proteins share similar percentage of identity with Slc7ala and Slc7a1b proteins, e.g., with respect to human SLC7A1, these values span from $\sim 71.5$ to $\sim 67.3 \%$ for Slc7ala and from 73.5 to $\sim 66.8 \%$ for Slc $7 \mathrm{a} 1 \mathrm{~b}$ transporters (for details, see Fig. S2). Therefore, also the identity criterion does not allow defining orthology between SLC7A1/Slc7a1 and SLc7a1a or Slc7alb proteins.

Only additional comparison at the genomic level shows the (slight) syntenic conservation of the zebrafish, and teleost fish, slc7ala gene with respect to the higher vertebrate slc7a1/Slc7a1/SLC7A1 series. In fact, slc7ala shares neighborhood with $m t u s 2 a$ in teleost fish while slc7a1/Slc7a1/SLC7A1 genes in amphibians, birds, and mammals do with mtus2/Mtus2/MTUS2.

All together, these conserved features strongly support the authenticity of the predicted zebrafish Slc7ala and put it in the orthologous line of the slc7a1/Slc7a1/ SLC7A1 series of higher vertebrates.

Notably, beside the already mentioned $\mathrm{E}^{107}$ residue within the third TM domain, our sequence analysis identified a special feature represented by a set of conserved negatively and positively charged amino acids (e.g., $\mathrm{R}^{362}, \mathrm{D}^{369}, \mathrm{D}^{370}, \mathrm{~K}^{375}, \mathrm{E}^{382}, \mathrm{R}^{383}, \mathrm{~T}^{384}$, and $\mathrm{K}^{385}$ ) observable within a larger region encompassing the fourth intracellular loop in the zebrafish Slc7ala isoform $\mathrm{X} 1$. This is substituted in isoform $\mathrm{X} 2$ by a similar stretch of amino acids but with differently charged amino acids in the parallel positions due to a splicing event involving exon $6 \mathrm{a}$ and exon $6 \mathrm{~b}$ (for details, see Fig. 1 and 4 and Fig. S1). Such a feature is typical of both teleost fish 

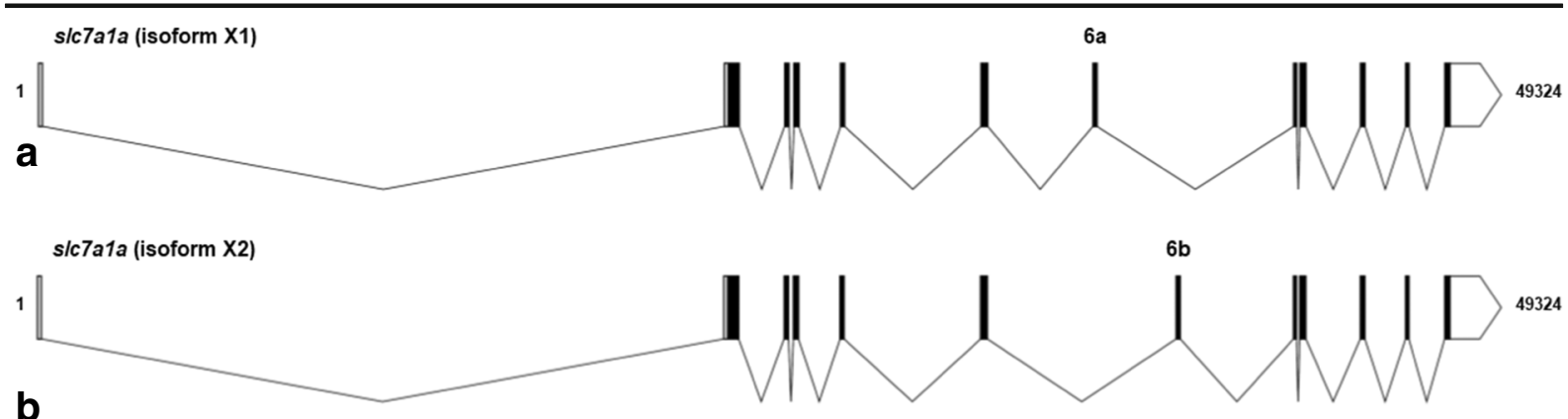

human_SLC7A1|NP_003036.1

SLLGSMFPMPRVIYAMAEDGLLFKFLANVNDRTKTPIIATLASGAVAA

SLLGSMFMPRVYYMAEDGLLFKFLAKVNDRTKTPIIATLASGAIAA

Cattle_SLC7A1|NP_D01129264.1

mouse_SlC7a1|NP_001288353.1

tropical clawed frog Slc7a1|XP 002934074.1

Atlantic_herring_Slc7a1a_isoform_X1|XP_012683156.1

SLLGSMFPMPRVIYAMAEDGLLFKFLAKVNDRTKKTPIIATLTSGAIAA

SLLGSMEPMPRVIYAMAEDGLEKFLAKINNRTKTPVIATVTSGAIAA

SLLGSMFPMPRIIYAMAEDGLLFKFLAKVNDKRKTPVIATVTSGAVAA

zebrafish Slc7a1a isoform X1|XP_683623.4 SLLGSMFPMPRVIFAMADDGLLFKFLAKVSEKTKTPVIATLTSGSVAA

SLLGAMFPMPRVIWAMAEDGLLFKYMADIHPRTKTPLLATLTSGIVAA SLLGAMFPMPRVLWAMADDGLLFKFMAGISERTKTPIKATIMSGFLAA

medaka Slc7a1a isoform X1 XP 011481652.1 SLLGAMFPMPRVIWAMADDGLLFKFMAEISPRTKTPLIATFASGTGAA

Atlantic herring Slc7a1b isoform X1|XP 031428872.1 SLLGSMFPMPRVIWAMAEDGLLFKFLANISPRTKTPIIATLTSGTVAA

zebrafish Slc7a1b isoform X1|NP 001315138.1 SLLGSMFPMPRVIWAMAEDGLLFKFLANISEKSKTPIMATVTSGIVAA

medaka Slc7a1

fugu_rubripes

fugu rubripes Slc7a1b isoform X3 XP 029699603.1 SLLVGMLPMPRVMWAMAKDGLLFKSLANISPRTKTPVAATLISGAWAA

human SLC7A3 INP 001041629.1 SLLGSMFPMPRVIYAMAEDGLLFRVLARIHTGTRTPIIATVVSGIIA

mouse_Slc7a3|NP_001288769.1 SLLGSMFPMPRVMYSMAEDGLLFRVLAKVHSVTHIPIVATLVSGVIA

SLLGSIFPMPRVIYAMAEDGLLFKCLAQINSKTKTPIIATLSSGAVAA

\begin{tabular}{ll} 
mouse Cat-2b|XP 006509313.1 & SLLGSIFPMPRVIYAMAEDGLLFKCLAOINSKTKTPVIATLSSGAVAA \\
\hline Atlantic herring Slc7a1a isoform X2|XP 012683159.1 & SLLGSMFPLPRIIFAMARDGLLFSYLARVSER-RTPIMSTMAAGAMSA
\end{tabular}

CAT-2B/Cat-2b-type (high affinity) proteins

$\begin{array}{ll}\text { Atlantic_herring_Slc7a1a_isoform_X2|XP_012683159.1 } & \text { SLLGSMFPLPRIIFAMARDGLLFSYLARVSER-RTPIMSTMAAGAMSA } \\ \text { zebrafish_Slc7a1a_isoform_X2|XP_005155334.1 } & \text { SLLGSLFPLPRIIFAMARDGLLFSFLARVSEK-KTPVVSTLASGVTAA }\end{array}$

$\begin{array}{ll}\text { zebrafish_Slc7a1a_isoform_X2|XP_005155334.1 } & \text { SLLGSLFPLPRIIFAMARDGLLFSFLARVSEK-KTPVVSTLASGVTAA } \\ \text { medaka_Slc7a1a_isoform_X2|XP_011481655.1 } & \text { SLLGSMFPLPRVIFAMARDGLLFSFLARISER-KAPVNSTVAAGVMSA }\end{array}$

fugu rubripes Slc7a1a X $11618220.1 \quad$ SLLGSMFPLPRIIYAMARDGLLFSFLARISER-KSPVTSTVTAGVMSA

Atlantic_herring_Slc7a1b_isoform_X2|XP_012686374.2 SLLGSMFPLPRIIYAMARDGLLFSFLARVSER-KTPMVATAASGFMSA

zebrafish_Slc7a1b_isoform_X2|XP_ô213370̄047.1 SLLGSMFPLPRIIFAMANDGLIFSFLSRVSER-KTPIVATTGAGLLSA

medaka_Slc7a1b_isoform_X2|XP_020563769.1 SLLGSMFPLPRIIFAMARDGLLFSFLAHVSER-KTPTVSTLVAGLMSA

fugu rubripes Slc7a1b isoform X2|XP 029699602.1 SLLGSMFPLPRIIFAMARDGLLYSFLARVSER-KTPILSTMVAGLLSA

human_CAT-2A|AAB62810.1 SLLGSMFPLPRILFAMARDGLLFRFLARVSKR-QSPVAATLTAGVISA

mouse Cat-2al XP 006509314.1 SLLGSMFPLPRILFAMARDGLLFRFLARVSKR-QSPVAATMTAGVISA

CAT-2A/Cat-2a-type (low affinity) proteins

\begin{tabular}{l} 
GKApcT $|\mathrm{pdb}| 500 \mathrm{VLLVMMYGQTRLFYAISRDGLLPKVFARISPTRQVPYVNTWLTGAAVA}$ \\
\hline
\end{tabular}

Bacterial CAT homologue (low affinity protein)

\section{C}

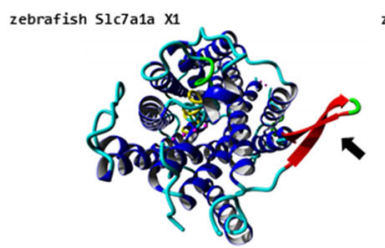

zebrafish S1c7a1a X2

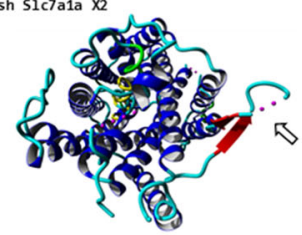

zebrafish S1c7a2 (Cat-2a)

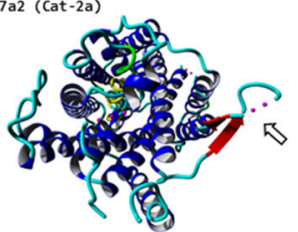

zebrafish Slc7a2 (Cat-2b)
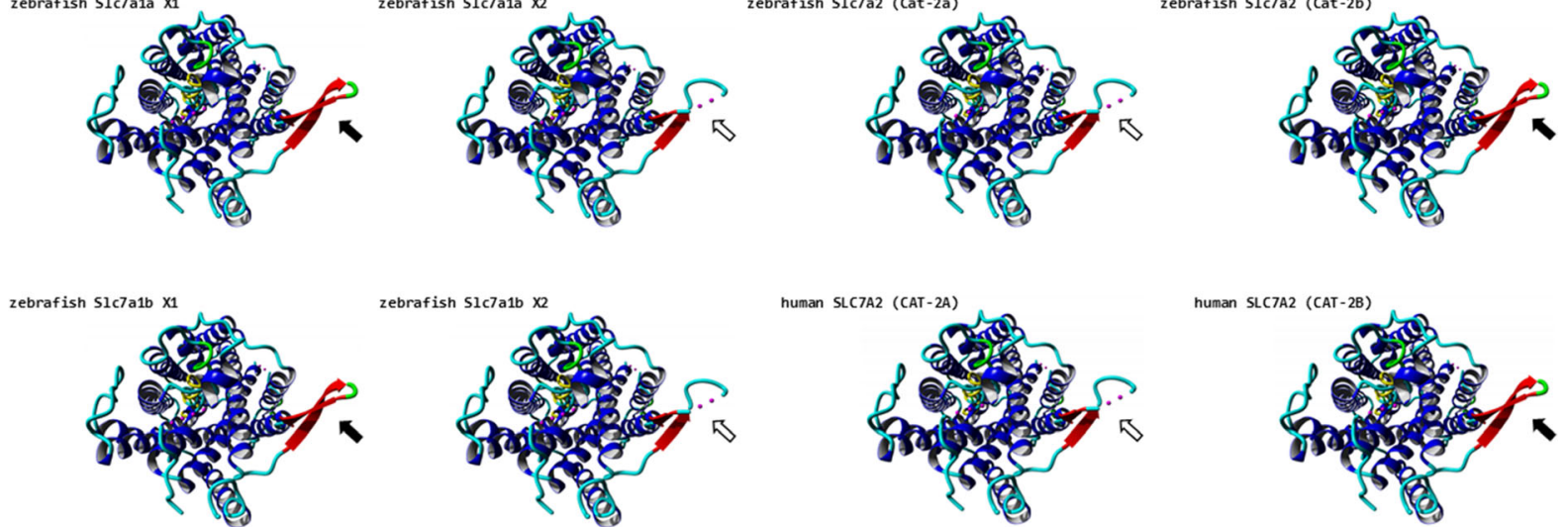

zebrafish 51c7a1b X2
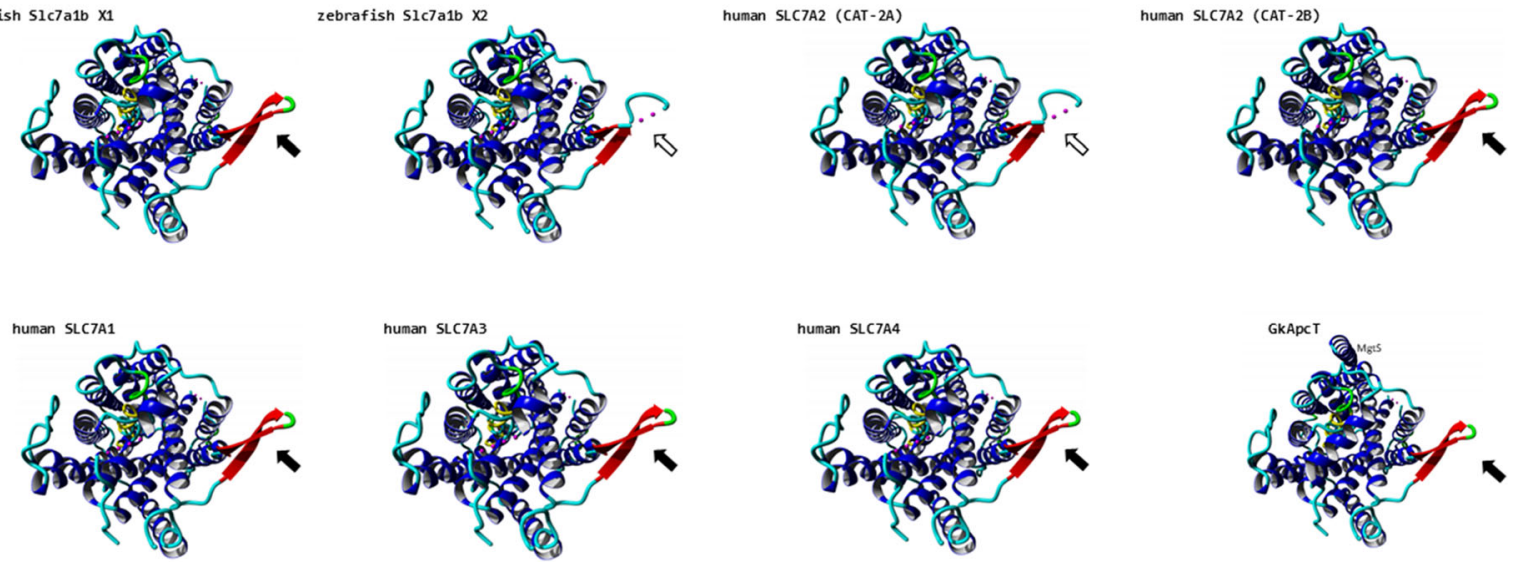
4 Fig. 4 a Schematic diagram depicting the genomic (exon-intron) organization of the zebrafish slc7ala gene. The zebrafish slc7ala sequence is 49,324 nucleotides long. The alternative coding exons $6 \mathrm{a}$ and $6 \mathrm{~b}$ are specifically indicated. Coding exon regions are in black, non-coding exon (5'- and 3 '-untranslated) regions are in white (please note that a fully non-coding exon occurs upstream the zebrafish slc7ala coding exon 1), and intron regions are as broken lines connecting exons. b Comparison of coding exon 6's predicted amino acid sequences of teleost fish (Atlantic herring, zebrafish, medaka, fugu rubripes) Slc7ala and Slc7alb, amphibian (tropical clawed frog) Slc7a1, bird (chicken) SLC7A1, mammalian (human, rhesus monkey, cattle, mouse) Slc7a1/SLC7A1, mammalian (human, mouse) Slc7a2/SLC7A2, and mammalian (human, mouse) Slc7a3/SLC7A3 proteins. Multiple sequence alignment was generated using Clustal Omega at https://www.ebi.ac.uk/Tools/msa/clustalo/ using default parameters (for details, see also Fig. 1 and Fig. S1). GkApcT refers to the recently identified and characterized proton-coupled amino acid transporter with the leucine transporter (LeuT) fold from Geobacillus kaustophilus (Jungnickel et al. 2018; Wu et al. 2019). An upwards arrow marks an amino acid position within this region acknowledged to be relevant for the definition of the low vs. high substrate affinity state of the transporter. An arginine (R) associates with a low affinity state (see, e.g., Closs et al. 1997; Habermeier et al. 2003; Jungnickel et al. 2018; Wu et al. 2019). c Three-dimensional appearance (cytoplasmic view) of zebrafish Slc7ala proteins (isoforms X1 and X2) and comparison with zebrafish Slc7alb (isoforms $\mathrm{X} 1$ and $\mathrm{X} 2$ ) and Slc7a2 (Cat-2a and Cat-2b) and human SLC7A1, SLC7A2 (CAT-2A and CAT-2B), SLC7A3, and SLC7A4. Homology models as from Phyre $^{2}$ (template: GkApcT) and visualization by YASARA (see also Fig. 1c). For each protein, confidence value was $100 \%$ and the residues in the model covered $70-71 \%$ of the corresponding experimental structure. The region of alternative splicing spans from approximately the second half of transmembrane domain 8 to the first half of transmembrane domain 9 through the cytoplasmic amino beta hairpin loop (see arrows). White arrows denote those proteins for which low substrate affinity has been ascertained (human CAT-2A) (see, e.g., Closs et al. 1997; Habermeier et al. 2003) or can be hypothesized (zebrafish Slc7a1a isoform X2, zebrafish Slc7a1b isoform X2 and zebrafish Cat-2a). MgtS indicates a small protein (31 amino acids; a single transmembrane domain) that complexes with GkApcT (Jungnickel et al. 2018; Wu et al. 2019)

Slc7a1a and Slc7alb proteins. And this strongly brings such teleost fish protein types closer to the vertebrate SLC7A2/SLc7a2 proteins for the commonality of the splicing event that imparts the characteristics of high/ low affinity toward cationic amino acids (and possibly sensitivity to trans-stimulation) to human SLC7A2 and mouse Slc7a2 (Closs et al. 1997; Habermeier et al. 2003). These findings are summarized in Fig. 4, which depicts the alternative splicing exons in the zebrafish slc7ala gene structure (Fig. 4a), the alternative amino acid sequences in the zebrafish Slc7ala isoforms X1 and X2 (Fig. 4b), and the structural differences that emerge between them by comparison after homology models analysis (Fig. 4c). Evident are, e.g., the differences in the structural organization of the hairpin loop between transmembrane domains 8 and 9 , as evident is that the alternative sequences (Fig. 4b) and structures (Fig. 4c) of the zebrafish Slc7a1a proteins are typically shared by transporters for which low (i.e., mammalian CAT-2A) vs. high (i.e., mammalian CAT-2B) substrate affinity has been demonstrated. If confirmed functionally, a structural-functional paradigm so far attributed to SLC7A2/Slc7a2 proteins only would be to extend to other Cat-type proteins, such as the teleost fish Slc7ala and Slc7a1b proteins.

Alignment also showed that the mammalian Nterminal peptide sequence motif (Met-Gly-Cys) is not conserved in the non-mammalian species. Downstream to this motif, there are several conserved basic residues along with two cysteines. In mouse, this conserved Nterminal motif along with the conserved basic residues of Slc7a1 has been shown to give a regulatory signal for transport into or retention inside of diverse cell membrane compartments (Ou and Silver 2003). Since this motif is absent but the downstream basic residues are conserved in teleost fish Slc7ala (zebrafish included), an alternative regulatory signal might be present in these proteins.

Slc7a1a expression in early eye, branchial arches, and muscle development might indicate a role in the collagen synthesis pathway

slc7ala expression was observed in the developing eyes and somites as early as $14 \mathrm{hpf}$, which also continued at the 24-hpf stage. Although no visual function is present at $14 \mathrm{hpf}$, the Slc7a1-mediated arginine transport at the inner blood retinal barrier in rat has been indicated as relevant in visual functions since it provides precursors to NO in the neural retina (Tomi et al. 2009). Moreover, arginine is a precursor of collagen, which is the most abundant protein in animals (Berisio et al. 2002; Shoulders and Raines 2009) and its different types are associated with particular tissues such as skin, bone, tendon, ligaments, branchial arches, cornea, cartilage, kidney, glomeruli, retina, intestine, and more (Berisio et al. 2002; Shoulders and Raines 2009). Furthermore, biochemical collagen precursors such as proline or hydroxyproline derive from arginine, while the essential 
amino acid lysine also forms the majority of collagen (Wittmann et al. 2005; Barbul 2008). In this regard, the "putative-to-date" transport of arginine and lysine via Slc7a1a would act as a rate-limiting step in providing precursors for collagen synthesis. In addition, studies in zebrafish have reported various collagen types to be involved in development of morphological structures (Akhtar et al. 2008; Bader et al. 2009; Gansner and Gitlin 2008; Huang et al. 2009; Le Guellec et al. 2004; Pagnon-Minot et al. 2008; Xiao and Baier 2007). More specifically, TGF- $\beta_{1}$ signaling has been implicated in collagen synthesis, where it stimulates proline and polyamine synthesis by upregulating arginine transport and metabolism mediated by a selective increase in SLC7A1 mRNA, ornithine decarboxylase, and ornithine aminotransferase (Durante et al. 2001). These findings would support a hypothesis that SLC7A1 might act in the collagen synthesis pathway required for the formation of eyes, branchial arches, and nephrons.

\section{Slc7a1a and kidney development}

In zebrafish, two nephrons function as pronephric kidneys during early life stages, in contrast to the situation in mammalian species where the kidneys contain thousands of nephrons (Drummond 2003). The zebrafish pronephros contains 8 distinct segments including two proximal tubule segments and two distal tubule segments, which are similar to those of the mammalian nephron (Wingert et al. 2007; Wingert and Davidson 2008). Tubular fluid flow through pronephros starts as early as $24 \mathrm{hpf}$ (Vasilyev et al. 2009), well before the formation of glomerulus, while glomerular filtration starts around 40 hpf (Drummond 2005; Vasilyev et al. 2009). The zebrafish pronephric nephrons form a closed system of blood filtration, tubular resorption, and fluid excretion (Drummond 2005). The primary function of the fish pronephros is osmoregulation (Drummond 2005). Without a functional kidney, zebrafish larvae die of gross edema since they are hyperosmotic animals that live in a very dilute environment (Drummond 2005). For instance, defects are reported in zebrafish morphants for nephrin and podocin (genes required for the development of pronephric podocyte cell structure) leading to defective proximal tubules and consequent pericardial edema (Kramer-Zucker et al. 2005).

In this study, slc7ala expression was first observed in the distal part of nephrons at $24 \mathrm{hpf}$ and was still maintained at $5 \mathrm{dpf}$. Previously, SLC7A1 localization has been reported to the basolateral membrane of the polarized kidney epithelial (MDCK and HEK) cells (Kizhatil and Albritton 2002), while a decrease in its expression was observed during endothelial cell dysfunction related to chronic renal failure (Schwartz et al. 2006). In addition, arginine, that is the sole precursor for NO, governs NO synthesis in renal epithelial cells (Schwartz et al. 2008). Because of such a fundamental function, it could be hypothesized that a "still-tobe-demonstrated functionally" Slc7a1a-mediated arginine transport is relevant for the formation and function of zebrafish nephrons. In effects, studies in adult mice have shown that the bilateral ureteral ligation increases glomerular arginine transport via Slc7al upregulation (Schwartz et al. 2008), while in chronic renal failure, arginine uptake is attenuated through modulation of Slc7a1 (Schwartz et al. 2006).

slc7ala expression pattern indicates a role in ion regulation in the gills

slc7ala expression started to appear in gill primordia at $3 \mathrm{dpf}$ and appeared stronger at $5 \mathrm{dpf}$. This is consistent with the previous observation that the zebrafish pharyngeal arches produce gill filament primordia at $3 \mathrm{dpf}$ (Kimmel et al. 1995; Rombough 2002). Ion regulation is the prime function of the gills before their gasexchange and $\mathrm{O}_{2}$-chemosensory pathways develop (Rombough 2002), and in teleost gills, ion regulation is strictly associated with mitochondria-rich cell (MRC) located in the inter-lamellar region of the gill filament epithelium (Jonz and Nurse 2008). As a consequence, freshwater teleosts mediate $\mathrm{Na}^{+}, \mathrm{Ca}^{2+}$, and $\mathrm{Cl}^{-}$uptake across the epithelium into the blood, while the saltwater teleosts mediate ion extrusion through MRCs (also called chloride cells) (Perry 1997; Marshall 2002; Evans et al. 2005; Jonz and Nurse 2008). Various neurotransmitters and neuropeptides, such as NO (eventually derived from arginine), catecholamines, acetylcholine, vasoactive intestinal polypeptide, endothelin, and prostaglandins, have been shown to mediate the movement of ions across gill and opercular epithelia, and stimulation of $\mathrm{Cl}^{-}$transport is mediated by adrenergic receptors (Marshall 2002; Evans et al. 2004; Evans et al. 2005; Jonz and Nurse 2008). Considering this information, we conclude that Slc7ala, being a source of arginine (precursor to NO), may be involved in gill ion regulation. 
Slc7a1a might be involved in ammonia excretion

It was originally considered that ammonia and urea move passively through tissues along partial pressure or concentration gradients. It is now proved that it requires Rhesus $(\mathrm{Rh})$ proteins for ammonia transport (Marini et al. 1997) and urea transporter proteins (UT) for urea to efficiently cross plasma membranes (Levine et al. 1973; You et al. 1993; Shayakul et al. 1996; Braun et al. 2009b). We found slc7ala to be expressed in the distal tubule segment of the nephron from $24 \mathrm{hpf}$ and onwards, while it was expressed in the gills from $3 \mathrm{dpf}$. There is compelling evidence in animals (fish included) that, unlike storage of excess lipids and carbohydrate, the excess of amino acids and proteins is usually metabolized to ammonia (Wright 1995; Terjesen et al. 2002; Braun et al. 2009a). We propose that Slc7alamediated transport may also be involved in the amino acid metabolism through the excretion of amino acid waste products such as ammonia and urea through kidney and gills. It has been reported that most teleost fishes excrete nitrogenous waste as ammonia through gills without converting it to urea or uric acid while freshwater fish can also excrete ammonia into urine to maintain the body fluid's acid-base balance at low external pH (King and Goldstein 1983; Wood et al. 1999; Nakada et al. 2007). Notably, the ammonia transporter Rhcg1 was shown to be localized in apical MRCs of yolk sac, gill, and particularly in the distal tubules of the zebrafish nephron (Nakada et al. 2007). In addition, the knockdown of ammonia (Rhag, Rhbg, and Rhcg1) or urea transporters (UT) in developing zebrafish has been shown to reduce the ammonia and urea excretion (Braun et al. 2009a). Interestingly, the same study reported an expression of these ammonia and urea transporters in the gills and distal nephron of the 4-dpf zebrafish larvae (Braun et al. 2009a), which is similar to our observation of slc7ala expression. Taken together, these findings suggest a possible link between the Slc7ala (an amino acid transporter) and the Rh and UT (ammonia and urea transporter) in the amino acid metabolism. However, detailed co-localization and functional studies are essential to establish this link.

\section{Conclusion and perspectives}

This study clarifies the gene/genomic organization of the zebrafish slc7ala gene in the context of teleost fish Cat-type transporters and suggests the existence of a much more complex set up and regulation of Cat-type genes in zebrafish, and teleost fish genomes, with respect to higher vertebrates. Our study also shows the specific expression pattern of slc7ala during zebrafish embryonic development suggesting the possible involvement in development and function of the eyes, kidney, muscles, and gills. The possible link between Slc7ala-mediated cationic amino acid transport/ homeostasis and ionoregulation and ammonia/urea transport, as well as other general physiological processes, needs to be established in zebrafish.

Acknowledgments We thank Heikki Savolainen and Grigory Merkin for taking very good care of our zebrafish.

Authors' contributions SN, IR, AF, and SE conceived the project and planned the experiments. SN performed experiments. All authors discussed the results, SN prepared figures, and TV prepared figures and tables. All authors wrote the manuscript, read, and approved the final manuscript.

Funding Open Access funding provided by University of Bergen. This work was financed by the Research Council of Norway (RCN) Strategic University Program Project 174979/I30. It also received support from RCN project 311627, University of Bergen (Projects NettMett and the Meltzer foundation 2020), University of Salento (Fondi ex-60\%), and the Apulian Region (Project 78M4CM5).

Data availability The authors confirm that the data supporting the findings of this study are available within the article and its supplementary materials.

Compliance with ethical standards Zebrafish were maintained and experiments conducted in compliance with the Norwegian Animal Welfare Act guidelines. No ethical permission was needed. According to the EU Directive 2010/63/EU on the protection of animals used for scientific purposes, imple

Conflict of interest The authors declare that they have no conflict of interest.

Open Access This article is licensed under a Creative Commons Attribution 4.0 International License, which permits use, sharing, adaptation, distribution and reproduction in any medium or format, as long as you give appropriate credit to the original author(s) and the source, provide a link to the Creative Commons licence, and indicate if changes were made. The images or other third party material in this article are included in the article's Creative Commons licence, unless indicated otherwise in a credit line to the material. If material is not included in the article's Creative Commons licence and your intended use is not permitted by statutory regulation or exceeds the permitted use, you will need to obtain 
permission directly from the copyright holder. To view a copy of this licence, visit http://creativecommons.org/licenses/by/4.0/.

\section{References}

Akhtar S, Schonthaler HB, Bron AJ, Dahm R (2008) Formation of stromal collagen fibrils and proteoglycans in the developing zebrafish cornea. Acta Ophthalmol 86:655-665

Albritton LM, Tseng L, Scadden D, Cunningham JM (1989) A putative murine ecotropic retrovirus receptor gene encodes a multiple membrane-spanning protein and confers susceptibility to virus infection. Cell 57:659-666

Albritton LM, Kim JW, Tseng L, Cunningham JM (1993) Envelope-binding domain in the cationic amino acid transporter determines the host range of ecotropic murine retroviruses. J Virol 67:2091-2096

Alexander SPH, Kelly E, Mathie A, Peters JA, Veale EL, Armstrong JF, Faccenda E, Harding SD, Pawson AJ, Sharman JL, Southan C, Davies JA, CGTP Collaborators (2019) The concise guide to pharmacology 2019/20: transporters. Br J Pharmacol 176(Suppl 1):S397-S493

Bader HL, Keene DR, Charvet B, Veit G, Driever W, Koch M, Ruggiero F (2009) Zebrafish collagen XII is present in embryonic connective tissue sheaths (fascia) and basement membranes. Matrix Biol 28:32-43

Barbul A (2008) Proline precursors to sustain mammalian collagen synthesis. J Nutr 138:2021S-2024S

Berisio R, Vitagliano L, Mazzarella L, Zagari A (2002) Recent progress on collagen triple helix structure, stability and assembly. Protein Pept Lett 9:107-116

Braun MH, Steele SL, Ekker M, Perry SF (2009a) Nitrogen excretion in developing zebrafish (Danio rerio): a role for Rh proteins and urea transporters. Am J Physiol Ren Physiol 296:F994-F1005

Braun MH, Steele SL, Perry SF (2009b) The responses of zebrafish (Danio rerio) to high external ammonia and urea transporter inhibition: nitrogen excretion and expression of rhesus glycoproteins and urea transporter proteins. J Exp Biol 212:3846-3856

Broer S (2008) Amino acid transport across mammalian intestinal and renal epithelia. Physiol Rev 88:249-286

Bronte V, Zanovello P (2005) Regulation of immune responses by L-arginine metabolism. Nat Rev Immunol 5:641-654

Cariappa R, Heath-Monnig E, Furesz TC, Kamath SG, Smith CH (2002) Stable polarized expression of hCAT-1 in an epithelial cell line. J Membr Biol 186:23-30

Civitelli R, Villareal DT, Agnusdei D, Nardi P, Avioli LV, Gennari C (1992) Dietary L-lysine and calcium metabolism in humans. Nutrition 8:400-405

Closs EI, Gräf P, Habermeier A, Cunningham JM, Förstermann U (1997) Human cationic amino acid transporters hCAT-1, hCAT-2A, and hCAT-2B: three related carriers with distinct transport properties. Biochemistry 36:6462-6468

Closs EI, Simon A, Vekony N, Rotmann A (2004) Plasma membrane transporters for arginine. J Nutr 134:2752S-2759S discussion 2765S-2767S
Closs EI, Boissel JP, Habermeier A, Rotmann A (2006) Structure and function of cationic amino acid transporters (CATs). J Membr Biol 213:67-77

Deves R, Boyd CA (1998) Transporters for cationic amino acids in animal cells: discovery, structure, and function. Physiol Rev 78:487-545

Deves R, Angelo S, Rojas AM (1998) System y+L: the broad scope and cation modulated amino acid transporter. Exp Physiol 83:211-220

Drummond I (2003) Making a zebrafish kidney: a tale of two tubes. Trends Cell Biol 13:357-365

Drummond IA (2005) Kidney development and disease in the zebrafish. J Am Soc Nephrol 16:299-304

Durante W, Liao L, Reyna SV, Peyton KJ, Schafer AI (2001) Transforming growth factor-beta(1) stimulates L-arginine transport and metabolism in vascular smooth muscle cells: role in polyamine and collagen synthesis. Circulation 103: $1121-1127$

Evans DH, Rose RE, Roeser JM, Stidham JD (2004) NaCl transport across the opercular epithelium of Fundulus heteroclitus is inhibited by an endothelin to NO, superoxide, and prostanoid signaling axis. Am J Phys Regul Integr Comp Phys 286: R560-R568

Evans DH, Piermarini PM, Choe KP (2005) The multifunctional fish gill: dominant site of gas exchange, osmoregulation, acid-base regulation, and excretion of nitrogenous waste. Physiol Rev 85:97-177

Felsenstein J (1985) Confidence limits on phylogenies: an approach using the bootstrap. Evolution 39:783-791

Fini M, Torricelli P, Giavaresi G, Carpi A, Nicolini A, Giardino R (2001) Effect of L-lysine and L-arginine on primary osteoblast cultures from normal and osteopenic rats. Biomed Pharmacother 55:213-220

Flodin NW (1997) The metabolic roles, pharmacology, and toxicology of lysine. J Am Coll Nutr 16:7-21

Gansner JM, Gitlin JD (2008) Essential role for the alpha 1 chain of type VIII collagen in zebrafish notochord formation. Dev Dyn 237:3715-3726

Gu Q, Yang X, Lin L, Li S, Li Q, Zhong S, Peng J, Cui Z (2014) Genetic ablation of solute carrier family 7a3a leads to hepatic steatosis in zebrafish during fasting. Hepatology 60:19291941

Habermeier A, Wolf S, Martine U, Graf P, Closs EI (2003) Two amino acid residues determine the low substrate affinity of human cationic amino acid transporter-2A. J Biol Chem 278: 19492-19499

Hatzoglou M, Fernandez J, Yaman I, Closs E (2004) Regulation of cationic amino acid transport: the story of the CAT-1 transporter. Annu Rev Nutr 24:377-399

Hediger MA, Clémençon B, Burrier RE, Bruford EA (2013) The $\mathrm{ABCs}$ of membrane transporters in health and disease (SLC series): introduction. Mol Asp Med 34:95-107

Huang CC, Wang TC, Lin BH, Wang YW, Johnson SL, Yu J (2009) Collagen IX is required for the integrity of collagen II fibrils and the regulation of vascular plexus formation in zebrafish caudal fins. Dev Biol 332:360-370

Jin ZB, Huang XF, Lv JN, Xiang L, Li DQ, Chen J, Huang C, Wu J, Lu F, Qu J (2014) SLC7A14 linked to autosomal recessive retinitis pigmentosa. Nat Commun 5:3517 
Jonz MG, Nurse CA (2008) New developments on gill innervation: insights from a model vertebrate. J Exp Biol 211:23712378

Jungnickel KEJ, Parker JL, Newstead S (2018) Structural basis for amino acid transport by the CAT family of SLC7 transporters. Nat Commun 9:550

Kandasamy P, Gyimesi G, Kanai Y, Hediger MA (2018) Amino acid transporters revisited: new views in health and disease. Trends Biochem Sci 43:752-789

Kelley LA, Mezulis S, Yates CM, Wass MN, Sternberg MJ (2015) The Phyre2 web portal for protein modeling, prediction and analysis. Nat Protoc 10:845-858

Kim JW, Cunningham JM (1993) N-linked glycosylation of the receptor for murine ecotropic retroviruses is altered in virusinfected cells. J Biol Chem 268:16316-16320

Kimmel CB, Ballard WW, Kimmel SR, Ullmann B, Schilling TF (1995) Stages of embryonic development of the zebrafish. Dev Dyn 203:253-310

King PA, Goldstein L (1983) Renal ammonia excretion and production in goldfish, Carassius auratus, at low environmental $\mathrm{pH}$. Am J Phys 245:R590-R599

Kizhatil K, Albritton LM (2002) System y+ localizes to different membrane subdomains in the basolateral plasma membrane of epithelial cells. Am J Phys Cell Phys 283:C1784-C1794

Kramer-Zucker AG, Wiessner S, Jensen AM, Drummond IA (2005) Organization of the pronephric filtration apparatus in zebrafish requires Nephrin, Podocin and the FERM domain protein mosaic eyes. Dev Biol 285:316-329

Krieger E, Vriend G (2014) YASARA view - molecular graphics for all devices - from smartphones to workstations. Bioinformatics 30:2981-2982

Kumar S, Stecher G, Li M, Knyaz C, Tamura K (2018) MEGA X: molecular evolutionary genetics analysis across computing platforms. Mol Biol Evol 35:1547-1549

Le Guellec D, Morvan-Dubois G, Sire JY (2004) Skin development in bony fish with particular emphasis on collagen deposition in the dermis of the zebrafish (Danio rerio). Int $\mathrm{J}$ Dev Biol 48:217-231

Levine S, Franki N, Hays RM (1973) A saturable, vasopressinsensitive carrier for urea and acetamide in the toad bladder epithelial cell. J Clin Invest 52:2083-2086

MacLeod CL (1996) Regulation of cationic amino acid transporter (CAT) gene expression. Biochem Soc Trans 24:846-852

Marini AM, Urrestarazu A, Beauwens R, Andre B (1997) The Rh (rhesus) blood group polypeptides are related to NH4+ transporters. Trends Biochem Sci 22:460-461

Marshall WS (2002) $\mathrm{Na}(+), \mathrm{Cl}(-), \mathrm{Ca}(2+)$ and $\mathrm{Zn}(2+)$ transport by fish gills: retrospective review and prospective synthesis. J Exp Zool 293:264-283

McDonald KK, Zharikov S, Block ER, Kilberg MS (1997) A caveolar complex between the cationic amino acid transporter 1 and endothelial nitric-oxide synthase may explain the "arginine paradox". J Biol Chem 272:31213-31216

Nakada T, Hoshijima K, Esaki M, Nagayoshi S, Kawakami K, Hirose S (2007) Localization of ammonia transporter Rhcg1 in mitochondrion-rich cells of yolk sac, gill, and kidney of zebrafish and its ionic strength-dependent expression. Am J Phys Regul Integr Comp Phys 293:R1743-R1753

Nguyen NTT, Vincens P, Roest Crollius H, Louis A (2018) Genomicus 2018: karyotype evolutionary trees and on-thefly synteny computing. Nucleic Acids Res 46:D816-D822
Nicholson B, Sawamura T, Masaki T, MacLeod CL (1998) Increased Cat3-mediated cationic amino acid transport functionally compensates in Cat1 knockout cell lines. J Biol Chem 273:14663-14666

North ML, Khanna N, Marsden PA, Grasemann H, Scott JA (2009) Functionally important role for arginase 1 in the airway hyperresponsiveness of asthma. Am J Phys Lung Cell Mol Phys 296:L911-L920

Ou W, Silver J (2003) Role of a conserved amino-terminal sequence in the ecotropic MLV receptor mCAT1. Virology 308:101-113

Pagnon-Minot A, Malbouyres M, Haftek-Terreau Z, Kim HR, Sasaki T, Thisse C, Thisse B, Ingham PW, Ruggiero F, Le Guellec D (2008) Collagen XV, a novel factor in zebrafish notochord differentiation and muscle development. Dev Biol 316:21-35

Perkins CP, Mar V, Shutter JR, del Castillo J, Danilenko DM, Medlock ES, Ponting IL, Graham M, Stark KL, Zuo Y, Cunningham JM, Bosselman RA (1997) Anemia and perinatal death result from loss of the murine ecotropic retrovirus receptor mCAT-1. Genes Dev 11:914-925

Perry SF (1997) The chloride cell: structure and function in the gills of freshwater fishes. Annu Rev Physiol 59:325-347

Rombough P (2002) Gills are needed for ionoregulation before they are needed for $\mathrm{O}_{2}$ uptake in developing zebrafish, Danio rerio. J Exp Biol 205:1787-1794

Rønnestad I, Murashita K, Kottra G, Jordal AE, Narawane S, Jolly C, Daniel H, Verri T (2010) Molecular cloning and functional expression of Atlantic salmon peptide transporter 1 in Xenopus oocytes reveals efficient intestinal uptake of lysine-containing and other bioactive di- and tripeptides in teleost fish. J Nutr 140:893-900

Saitou N, Nei M (1987) The neighbor-joining method: a new method for reconstructing phylogenetic trees. Mol Biol Evol 4:406-425

Schnorr O, Suschek CV, Kolb-Bachofen V (2003) The importance of cationic amino acid transporter expression in human skin. J Invest Dermatol 120:1016-1022

Schwartz F, Ayalon R, Chernichovski T, Reshef R, Chernin G, Weinstein T, Litvak A, Levo Y, Schwartz D (2006) Arginine uptake is attenuated through modulation of cationic aminoacid transporter-1, in uremic rats. Kidney Int 69:298-303

Schwartz IF, Davidovitz A, Chernichovski T, Levin-Iaina N, Guzner-Gur H, Levo Y, Schwartz D (2008) Arginine transport is augmented, through modulation of cationic amino acid transporter-1, in obstructive uropathy in rats. Kidney Blood Press Res 31:210-216

Seo HC, Drivenes Ø, Ellingsen S, Fjose A (1998) Expression of two zebrafish homologues of the murine Six 3 gene demarcates the initial eye primordia. Mech Dev 73:45-57

Shayakul C, Steel A, Hediger MA (1996) Molecular cloning and characterization of the vasopressin-regulated urea transporter of rat kidney collecting ducts. J Clin Invest 98:2580-2587

Shima Y, Maeda T, Aizawa S, Tsuboi I, Kobayashi D, Kato R, Tamai I (2006) L-arginine import via cationic amino acid transporter CAT1 is essential for both differentiation and proliferation of erythrocytes. Blood 107:1352-1356

Shoulders MD, Raines RT (2009) Collagen structure and stability. Annu Rev Biochem 78:929-958

Stuart GW, McMurray JV, Westerfield M (1988) Replication, integration and stable germ-line transmission of foreign 
sequences injected into early zebrafish embryos. Development 103:403-412

Terjesen BF, Finn RN, Norberg B, Rønnestad I (2002) Kinetics and fates of ammonia, urea, and uric acid during oocyte maturation and ontogeny of the Atlantic halibut (Hippoglossus hippoglossus L.). Comp Biochem Phys A 131:443-455

Thisse B, Thisse C (2004) Fast release clones: a high throughput expression analysis. ZFIN Direct Data Submission. http://zfin.org

Tomi M, Kitade N, Hirose S, Yokota N, Akanuma S, Tachikawa M, Hosoya K (2009) Cationic amino acid transporter 1mediated L-arginine transport at the inner blood-retinal barrier. J Neurochem 111:716-725

Umeki N, Fukasawa Y, Ohtsuki S, Hori S, Watanabe Y, Kohno Y, Terasaki T (2002) mRNA expression and amino acid transport characteristics of cultured human brain microvascular endothelial cells (hBME). Drug Metab Pharmacokinet 17: 367-373

Van Winkle LJ (2001) Amino acid transport regulation and early embryo development. Biol Reprod 64:1-12

Vasilyev A, Liu Y, Mudumana S, Mangos S, Lam PY, Majumdar A, Zhao J, Poon KL, Kondrychyn I, Korzh V, Drummond IA (2009) Collective cell migration drives morphogenesis of the kidney nephron. PLoS Biol 7:e9

Wang H, Kavanaugh MP, Kabat D (1994) A critical site in the cell surface receptor for ecotropic murine retroviruses required for amino acid transport but not for viral reception. Virology 202:1058-1060

Wang H, Klamo E, Kuhmann SE, Kozak SL, Kavanaugh MP, Kabat D (1996) Modulation of ecotropic murine retroviruses by N-linked glycosylation of the cell surface receptor/amino acid transporter. J Virol 70:6884-6891

Wingert RA, Davidson AJ (2008) The zebrafish pronephros: a model to study nephron segmentation. Kidney Int 73:11201127

Wingert RA, Selleck R, Yu J, Song HD, Chen Z, Song A, Zhou Y, Thisse B, Thisse C, McMahon AP, Davidson AJ (2007) The cdx genes and retinoic acid control the positioning and segmentation of the zebrafish pronephros. PLoS Genet 3:19221938

Wittmann F, Prix N, Mayr S, Angele P, Wichmann MW, van den Engel NK, Hernandez-Richter T, Chaudry IH, Jauch KW, Angele MK (2005) L-arginine improves wound healing after trauma-hemorrhage by increasing collagen synthesis. J Trauma 59:162-168

Wood CM, Milligan CL, Walsh PJ (1999) Renal responses of trout to chronic respiratory and metabolic acidoses and metabolic alkalosis. Am J Phys 277:R482-R492

Wright PA (1995) Nitrogen excretion: three end products, many physiological roles. J Exp Biol 198:273-281

Wu G, Bazer FW, Davis TA, Kim SW, Li P, Marc Rhoads J, Carey Satterfield M, Smith SB, Spencer TE, Yin Y (2009) Arginine metabolism and nutrition in growth, health and disease. Amino Acids 37:153-168

Wu Z, Alibay I, Newstead S, Biggin PC (2019) Proton control of transitions in an amino acid transporter. Biophys J 117:13421351

Xiao T, Baier H (2007) Lamina-specific axonal projections in the zebrafish tectum require the type IV collagen Dragnet. Nat Neurosci 10:1529-1537

You G, Smith CP, Kanai Y, Lee WS, Stelzner M, Hediger MA (1993) Cloning and characterization of the vasopressinregulated urea transporter. Nature 365:844-847

Zhang Y, Skolnick J (2005) TM-align: a protein structure alignment algorithm based on the TM-score. Nucleic Acids Res 33:2302-2309

Zuckerkandl E, Pauling L (1965) Evolutionary divergence and convergence in proteins. In: Edited in Evolving genes and proteins by V. Bryson and H.J. Vogel. Academic, New York, pp $97-166$

Publisher's note Springer Nature remains neutral with regard to jurisdictional claims in published maps and institutional affiliations. 